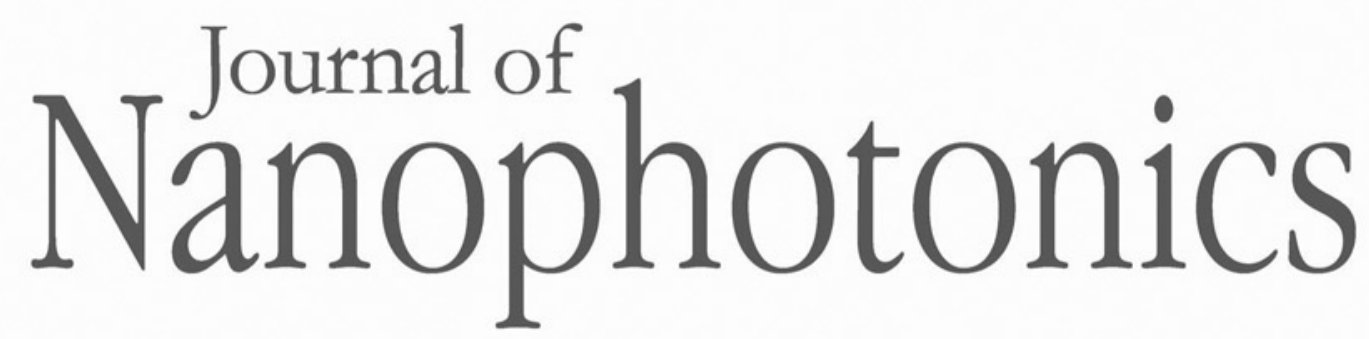

Nanophotonics.SPIEDigitalLibrary.org

\title{
Review of mid-infrared plasmonic materials
}

\author{
Yujun Zhong \\ Shyamala Devi Malagari \\ Travis Hamilton \\ Daniel Wasserman
}

\section{SPIE.}




\title{
Review of mid-infrared plasmonic materials
}

\author{
Yujun Zhong, Shyamala Devi Malagari, Travis Hamilton, and \\ Daniel Wasserman*
}

University of Illinois Urbana Champaign, Micro and Nanotechnology Lab, Department of Electrical Engineering, 208 North Wright Street, Urbana, Illinois 61822, United States

\begin{abstract}
The field of plasmonics has the potential to enable unique applications in the midinfrared (IR) wavelength range. However, as is the case regardless of wavelength, the choice of plasmonic material has significant implications for the ultimate utility of any plasmonic device or structure. In this manuscript, we review the wide range of available plasmonic and phononic materials for mid-IR wavelengths, looking in particular at transition metal nitrides, transparent conducting oxides, silicides, doped semiconductors, and even newer plasmonic materials such as graphene. We also include in our survey materials with strong mid-IR phonon resonances, such as $\mathrm{GaN}, \mathrm{GaP}, \mathrm{SiC}$, and the perovskite $\mathrm{SrTiO}_{3}$, all of which can support plasmon-like modes over limited wavelength ranges. We will discuss the suitability of each of these plasmonic and phononic materials, as well as the more traditional noble metals for a range of structures and applications and will discuss the potential and limitations of alternative plasmonic materials at these IR wavelengths. (C) 2015 Society of Photo-Optical Instrumentation Engineers (SPIE) [DOI: 10 .1117/1.JNP.9.093791]
\end{abstract}

Keywords: mid-infrared; plasmonics; optical materials; optics; photonics.

Paper 14145SS received Nov. 7, 2014; accepted for publication Dec. 30, 2014; published online Feb. 3, 2015.

\section{Introduction}

The field of plasmonics has grown over the past decades to encompass a wide range of phenomena and potential applications across a broad range of optical frequencies. At its most fundamental, the field of plasmonics can be described as the study of plasmons, or collective charge oscillations, in conducting or even semiconducting materials. In most instances, it is the interaction of these collective charge oscillations with electromagnetic radiation at the surface of the conducting material which leads to the phenomena most responsible for the ever-increasing interest in the field of plasmonics. These hybrid excitations are typically referred to as surface plasmons, and at a given frequency, with the proper choice of conducting material and surrounding dielectric, allow for the subwavelength confinement of optical modes. The structures and materials that support these surface plasmon excitations may play a key role in next-generation optical interconnects, optical sensing technologies, photothermal therapies and purification processes, as well as the emerging fields of nanophotonics, metamaterials and metasurfaces. From a more fundamental point of view, the strong electric fields associated with the subwavelength confinement of electromagnetic radiation can provide a test-bed for investigation of the lightmatter interaction in nanoscopic systems.

The pull of nanophotonics is such that the vast majority of plasmonics research has focused on the shorter wavelength side of the optical frequency range. It is here that the subwavelength confinement of optical modes can be achieved with nanoplasmonic structures, which have been hailed as a potential link between the nanoscale of modern day electronics and the approximate micron scale of optical communication wavelengths, as well as a potential path toward the development of optical sensors on the length scale of the molecules to be sensed. It is thus only natural that efforts to achieve optical confinement on the nanoscale would utilize shorter wavelength

*Address all correspondence to: D. Wasserman, E-mail: dwass@illinois.edu

0091-3286/2015/\$25.00 (C) 2015 SPIE 
light. However, there also exist a large number of potential applications for plasmonics at longer wavelengths, in particular, in the mid-infrared (mid-IR) wavelength range ( 3 to $30 \mu \mathrm{m}$ ), where many molecules have strong and distinct fundamental vibrational and rotational absorption lines and where the peak blackbody emission for most biological and mechanical objects can be found. Most short-wavelength plasmonic sensor systems rely on detecting a spectral shift in a plasmonic resonance, resulting from the change in the local index of refraction when the analyte of interest binds to a functionalized plasmonic structure. ${ }^{1,2}$ In the mid-IR, a similar system might allow for a direct detection of the analyte in question by directly probing the vibro-rotational absorption resonances of the molecule, without relying on the chemical differentiation associated with functionalized surfaces. Alternatively, plasmonic structures might enable the miniaturization of mid-IR optoelectronic devices, allowing for efficient confinement of subwavelength modes in mid-IR lasers, and the efficient coupling of free-space light to (from) subwavelength mid-IR detectors (sources). Plasmonic structures designed to selectively absorb light (and thus selectively emit thermal radiation upon heating) could serve as either narrow-band sources of IR radiation, or alternatively, as thermal coatings designed to alter the natural black body signal of the underlying object. Finally, the mid-IR also serves as an ideal testbed for next-generation plasmonic architectures, where engineered plasmonic metals can be integrated with a range of loss-less dielectrics as well as engineered quantum structures, to explore the ultimate limits of optoelectronic/plasmonic devices and architectures.

However, despite all of the promise of plasmonics, the continued development of plasmonic structures and devices for commercial applications has been limited. A number of plasmonic sensor systems have been commercialized, ${ }^{3}$ while plasmonic antennas have been used to decrease bit-size and thus increase storage density on hard drives using heat-assisted magnetic recording, ${ }^{4}$ and metallic nanoparticles have shown great promise in localized photothermal cancer therapies ${ }^{5}$ and nanoscale boiling without heating for water purification. ${ }^{6}$ Many of the most promising plasmonic technologies use the localized heating associated with surface plasmon resonances, effectively leveraging the inherent losses in plasmonic materials. However, few of the promised applications based on enhanced light-matter interaction have progressed past the research lab, due for the most part to the very same losses which are leveraged for the localized heating applications. There is a growing recognition that in order to realize the full potential of the field of plasmonics, a renewed emphasis must be placed on the engineering and development of plasmonic materials. ${ }^{7,8}$ One particularly striking example of this renewed emphasis on materials is the recent demonstration of epitaxially grown silver, and its application to plasmonic nanolasers. ${ }^{9}$ However, it is unlikely that a single silver (plasmonic?) bullet exists that will provide the ideal metal for the wide range of plasmonic applications across the wide range of optical frequencies. Instead, each potential application or plasmonic structure will require a careful choice of plasmonic material, and this choice of material will have to be made taking into consideration a number of factors. In this review article, we examine the potential plasmonic materials available for mid-IR photonic applications. Though plasmonic structures can assume an infinite variety of geometries, here we focus on a number of representative geometries, so that the relative performance of the plasmonic materials considered for each geometry can be used to extrapolate the performance of these materials for similar structures. For each plasmonic structure investigated, we will, when possible, provide a figure of merit (FOM) for each plasmonic material used in that geometry. In addition, we will discuss other potential benefits and challenges for each of the materials investigated, including cost, wavelength flexibility, tunability, temperature performance, and compatibility with current optoelectronic or CMOS fabrication processes. In summary, we endeavor to present a comprehensive picture of the current state-of-the-art, as well as an outlook for the future, of mid-IR plasmonic materials.

\section{Fundamentals of Plasmonic Materials}

Plasmonic phenomena are a direct result of collective charge excitations in the plasmonic material. It is important to note that very similar phenomena can be achieved utilizing collective lattice vibrations (phonons instead of plasmons). Although not technically plasmonic in nature, these surface phonon modes have many of the same features as their plasmonic counterparts, and for the sake of completeness, will be covered in this review as well. The behavior of free carriers 
in a material can most simply be described using the Drude model, where we assume the free electrons in a material can be described by an effective mass $m^{*}$ and a charge $-e$. The polarization resulting from the motion of free carriers under an applied harmonic electric field can then be determined by solving a simple equation of motion for the free electrons, assuming a frequency-independent scattering rate $\gamma$. The permittivity of the plasmonic material in the simple Drude model, $\varepsilon_{m}$, including the background permittivity from the bound electrons in the crystal, $\varepsilon_{b}$, becomes

$$
\varepsilon_{m}=\varepsilon_{m}^{\prime}+i \varepsilon_{m}^{\prime \prime}=\varepsilon_{b}-\frac{\omega_{p}^{\prime 2}}{\omega(\omega+i \gamma)}, \quad \omega_{p}^{\prime 2}=\frac{e^{2} n}{m^{*} \varepsilon_{o}},
$$

where $\varepsilon_{o}$ is the permittivity of free space, $\omega_{p}^{\prime}$ is the material plasma frequency, and $n$ is the freecarrier concentration. If the material is treated as a gas of free carriers $\left(\varepsilon_{b}=1\right)$, the plasma frequency corresponds to the frequency where the permittivity of the material is vanishingly small. For materials with substantial permittivity contributions from the polarizability of bound electrons, we can write the plasma frequency $\left(\omega_{p}^{2}\right)$ normalized to the background permittivity (as we will do for the remainder of our analysis) and retain the same physical meaning for the plasma frequency (the frequency where $\varepsilon_{m}^{\prime}$ is vanishingly small).

$$
\varepsilon_{m}=\varepsilon_{b}\left[1-\frac{\omega_{p}^{2}}{\omega(\omega+i \gamma)}\right], \quad \omega_{p}^{2}=\frac{e^{2} n}{m^{*} \varepsilon_{o} \varepsilon_{b}} .
$$

While many mid-IR plasmonic materials can be quite accurately modeled using the Drude approach, this model does not take into account several important phenomena which can cause the material's experimental permittivity to deviate from the above description. First, while the free carrier scattering can often be approximated as constant across a wide range of frequencies, a fully accurate representation of the permittivity would require a frequency-dependent scattering term $\gamma(\omega)$. As a simple and extreme example, it is well known that the DC conductivity of a metal is not necessarily a good indicator of the metal's plasmonic performance at optical frequencies, due to the frequency dependence of the material's scattering term. ${ }^{8}$ Second, the simple model above ignores the effects of absorption from interband transitions in the crystal bandstructure, each of which can be modeled as a Lorentzian absorption term. Thus, a more accurate model of the metal permittivity would be

$$
\varepsilon_{m}=\varepsilon_{b}\left\{1-\frac{\omega_{p}^{2}}{\omega\left[\omega+i \gamma_{D}(\omega)\right]}\right\}+\sum_{n} \frac{f_{n} \omega_{n}^{2}}{\omega_{n}^{2}-\omega^{2}+i \omega \gamma_{n}},
$$

where the interband absorption is modeled as a collection of Lorentzian absorption features, each with amplitude, central frequency, and broadening terms $f_{n}, \omega_{n}$, and $\gamma_{n}$, respectively.

It is ultimately the plasmonic metal's permittivity (both real and imaginary) which determines whether, and how well, the material can support a plasmonic mode. As a general rule, the real part of the plasmonic material's permittivity must be negative (though how negative depends on the desired plasmonic applications) and the imaginary part small. Once these conditions are met, the solution for the optical mode bound to the plasmonic material/dielectric surface comes from the solution to Maxwell's equations, and the free carrier behavior is simply wrapped into the permittivity. In other words, Maxwell's equations are oblivious to the source of the plasmonic material's permittivity, and the optical response of any materials with equivalent permittivities will be, in many ways, identical.

This fact becomes important when we consider the effect of collective lattice vibrations, or phonons, on the permittivity of optical materials. Solving for the exact response of a crystal lattice to the incident electromagnetic radiation requires not only an understanding of the three-dimensional (3-D) crystal lattice but also a quantum mechanical description of the excitations themselves. However, the typical approach to understanding the phonon contribution to the optical permittivity of a material is phenomenological, with

$$
\varepsilon_{\text {phon. }}(\omega)=\varepsilon(\infty)+[\varepsilon(0)-\varepsilon(\infty)] \frac{\omega_{\mathrm{TO}}^{2}}{\omega_{\mathrm{TO}}^{2}-\omega^{2}+i \omega \gamma_{\mathrm{phon} .}}, \quad \sqrt{\frac{\varepsilon(0)}{\varepsilon(\infty)}}=\frac{\omega_{\mathrm{LO}}}{\omega_{\mathrm{TO}}},
$$



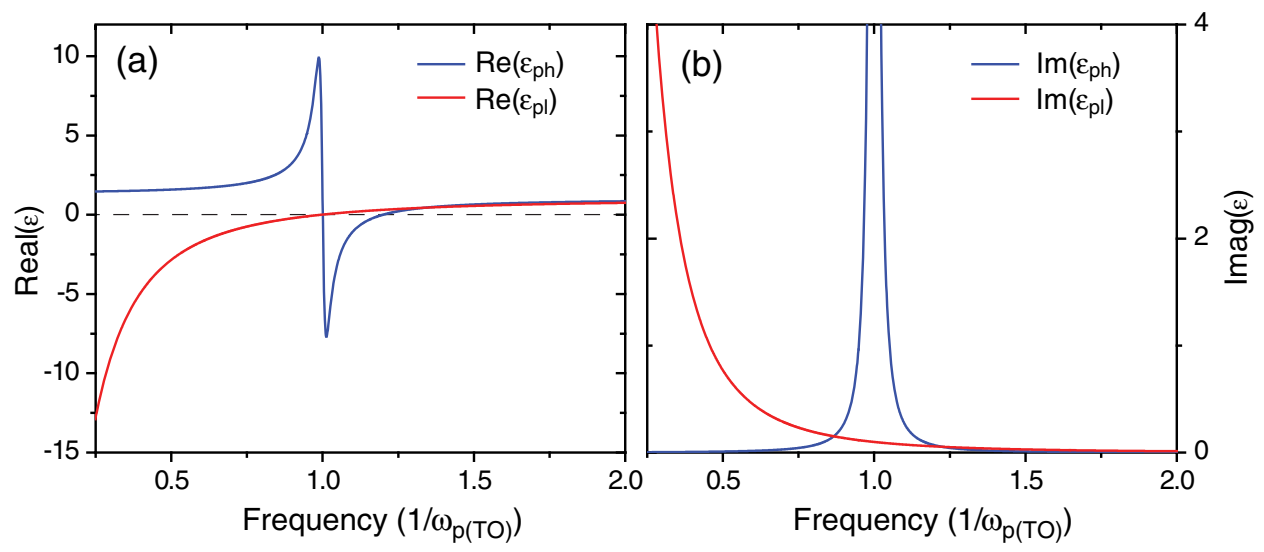

Fig. 1 Modeled (a) real and (b) imaginary components of the permittivity of a phononic (blue) and plasmonic (red) material. The frequency axes are dimensionless, normalized to the phononic and plasmonic materials' transverse optical phonon energy $\left(\omega_{\mathrm{TO}}\right)$ and plasma frequency $\left(\omega_{p}\right)$, respectively. For the modeled plasmonic material, we use Eq. 2, with $\varepsilon_{b}=1$, and $\gamma_{p l}=\omega_{p} / 10$, while for the phononic material we use Eq. 4 , with $\varepsilon(\infty)=1, \gamma_{p h}=\omega_{p} / 40$ and $\omega_{\text {LO }}=1.2 \omega_{\text {TO }}$.

where $\omega_{\mathrm{LO}}$ and $\omega_{\mathrm{TO}}$ are the longitudinal and transverse optical phonon energies, and $\varepsilon(0)$ and $\varepsilon(\infty)$ are the DC and high-frequency permittivity of the material, which are related above via the Lyddane-Sachs-Teller relation (which holds for diatomic cubic crystals). While the exact phonon-dependent permittivity is significantly more complex, the above expression (often with slight modification) provides an excellent fit for a wide range of materials. Figure 1 shows the real and imaginary permittivity for a "phononic" and "plasmonic" material. As can be seen from this figure, both materials exhibit negative permittivity. For the plasmonic material, modeled using the Drude-Lorentz formalism of Eq. (3), the permittivity is negative for frequencies lower than the plasma frequency $\left(\omega_{p}\right)$, while for the phononic material, modeled using the phenomenological expression given in Eq. (4), has negative permittivity only in the limited range of frequencies $\omega_{\mathrm{TO}}<\omega<\omega_{\mathrm{LO}}$. This illustrates a rather significant difference between phononic and plasmonic materials, namely that phononic materials can support surface modes only across a narrow range of frequencies determined by the materials' crystalline structure.

\section{Growth and Bulk Optical Properties of Mid-IR Plasmonic Materials}

In this work, we will investigate the potential of a wide range of materials for mid-IR plasmonic applications. We will begin with the traditional plasmonic materials most frequently used for visible and short wavelength IR applications, the noble metals. New classes of near-IR "designer" plasmonic materials, in particular, the transition metal nitrides and transparent conducting oxides, will also be discussed. However, the bulk of our efforts will focus on plasmonic materials with primary applications in the mid-IR. In this category, we include plasmonic materials compatible with optoelectronic and CMOS devices and fabrication processes, such as the silicides, and materials with plasma frequencies squarely in, or at least in close proximity to, the mid-IR portion of the electromagnetic spectrum, such as doped semiconductors. We will also discuss phononic materials, such as $\mathrm{SiC}, \mathrm{GaN}, \mathrm{GaP}$, and the Perovskite $\mathrm{SrTiO}_{3}$, whose high energy phonons allow for negative permittivity in the mid-IR. Finally, we will briefly discuss the relatively new field of two-dimensional (2-D) plasmonic materials, using graphene as our representative 2-D material.

Much of the earliest work in plasmonics utilized noble metals ( $\mathrm{Au}, \mathrm{Ag})$ to demonstrate plasmonic behavior in the visible (VIS) and near-infrared (near-IR) portion of the spectrum. For planar plasmonic structures, these metals are traditionally deposited via e-beam or thermal evaporation. Minimal depositions, below the so-called percolation threshold, form islands or discontinuous films, which can support localized plasmonic modes, ${ }^{10}$ and have been leveraged for sensing applications. ${ }^{11,12}$ Above the percolation threshold, the metal layer becomes effectively planar, though surface roughness and grain boundaries can increase losses from the 
ideal. Figure 2 shows both the experimental permittivity ${ }^{13}$ and the Drude model fit to the experimental data for three noble metals $(\mathrm{Ag}, \mathrm{Au}$, and $\mathrm{Cu})$ often used for plasmonic structures in the visible and near-IR frequency range. As can be seen in Fig. 2(a), these "traditional" plasmonic metals show plasma frequencies at visible frequencies (or higher, in the UV) as well as strong interband absorption features above, or in spectral proximity to, the plasma frequency. In the IR [as seen in Fig. 2(b)], all of the traditional plasmonic metals have extremely large, negative permittivity, an effect which will have significant repercussions for their utility in mid-IR plasmonic applications. Though we only show three metals in Fig. 2, it should be noted that the basic form of these metals' frequency-dependent permittivity is not only seen in the noble metals but also in the group III metals ( $\mathrm{Al}, \mathrm{Ga}$, In $)^{14-17}$ and the transition metals $(\mathrm{Cr}, \mathrm{Pd}, \mathrm{Pt}, \mathrm{Ni}, \mathrm{Ti}, \mathrm{W}) .^{18}$ Many of these metals react with the atmosphere and/or water, limiting their utility for a wide range of applications.

The UV/VIS frequency position of the plasma frequency for these metals can be easily understood by considering the large numbers of free electrons available to interact with incident radiation (Eq. 1). By "diluting" the free-carrier concentration of a metal, the metal plasma frequency can be shifted to longer wavelengths. Alternatively, one could begin with non-conducting insulators, and by doping these materials with increasing amounts of free carriers, shift the materials' plasma frequencies to shorter wavelengths. In practice, the end result of both of the above approaches is some form of "intermetallic." It is important to note that not only does such an approach allow for greater control of the material plasma frequency, but by controlling the material composition, one can hope to engineer the crystalline bandstructure and gain some modicum of control over the interband absorption features which serve as limiting factors for many plasmonic applications near a plasmonic materials' plasma frequency. Examples of intermetallics are the silicides and germanides, ${ }^{19-21}$ often formed by sequentially deposited semiconductors (silicon or germanium, respectively) and metals $(\mathrm{Cu}, \mathrm{Co}, \mathrm{Pd}, \mathrm{Pt}, \mathrm{Ni}, \mathrm{Ti}$, for example) followed by a high temperature anneal to form a conducting material. While the majority of silicide and germanide research has focused on integration into CMOS fabrication technology, such materials are also of particular interest for longer-wavelength plasmonic applications, ${ }^{22}$ due to their CMOS compatibility, and plasmonic behavior at frequencies below the band-edge of $\mathrm{Si}$ or Ge (and thus spectrally separated from interband absorption losses). Figure 3 shows the experimental and Drude model permittivity for representative silicide materials (NiSi and TiSi), from Ref. 22. As can be seen from the data in Fig. 3, these materials also have large negative real parts of the permittivity in the mid-IR. In addition to the germanides and silicides, alkali-noble intermetallics, conducting oxides and nitrides, which include materials such as indium-tin-oxide, aluminum- or gallium-doped zinc oxide (AZO and GZO, respectively), and transition-metal nitrides, all have significant potential as near-IR plasmonic materials. ${ }^{23-26}$ Conducting oxides,

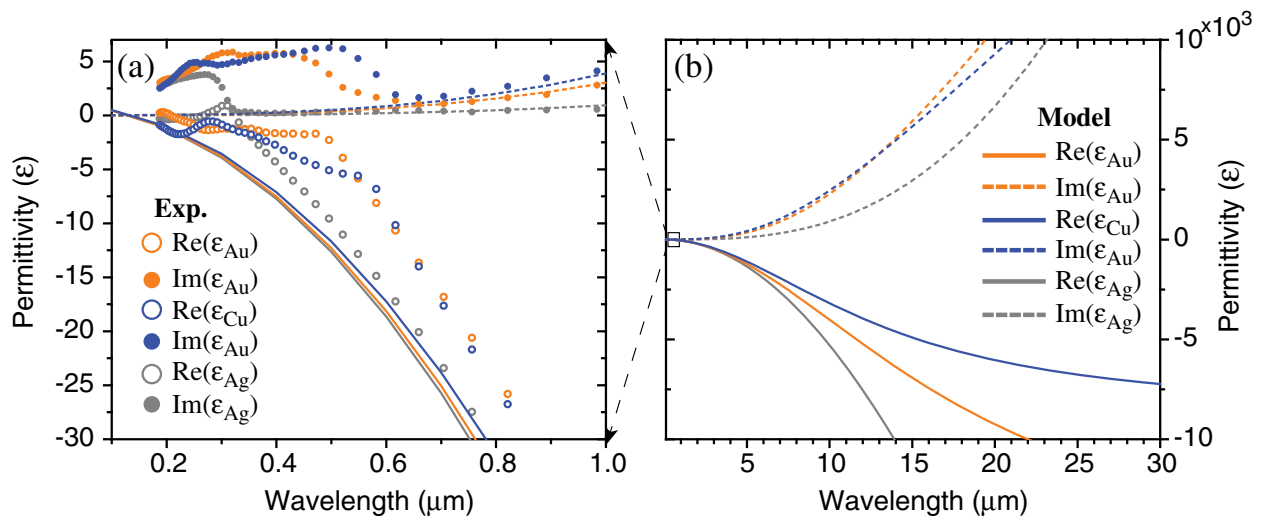

Fig. 2 (a) Permittivity at short wavelengths $(0.1-1 \mu \mathrm{m})$ for three noble metals: Ag (orange), Au (gray) and $\mathrm{Cu}$ (blue), showing both experimental data for the real (empty circle) and imaginary (filled circle) parts of the permittivity. (b) Drude model of real (solid) and imaginary (dashed) permittivity for the three noble metals in (a). Drude model permittivity is overlaid in (a) in order to demonstrate the deviation from the free electron model resulting from interband absorption (experimental data and Drude model parameters from Ref. 13). 


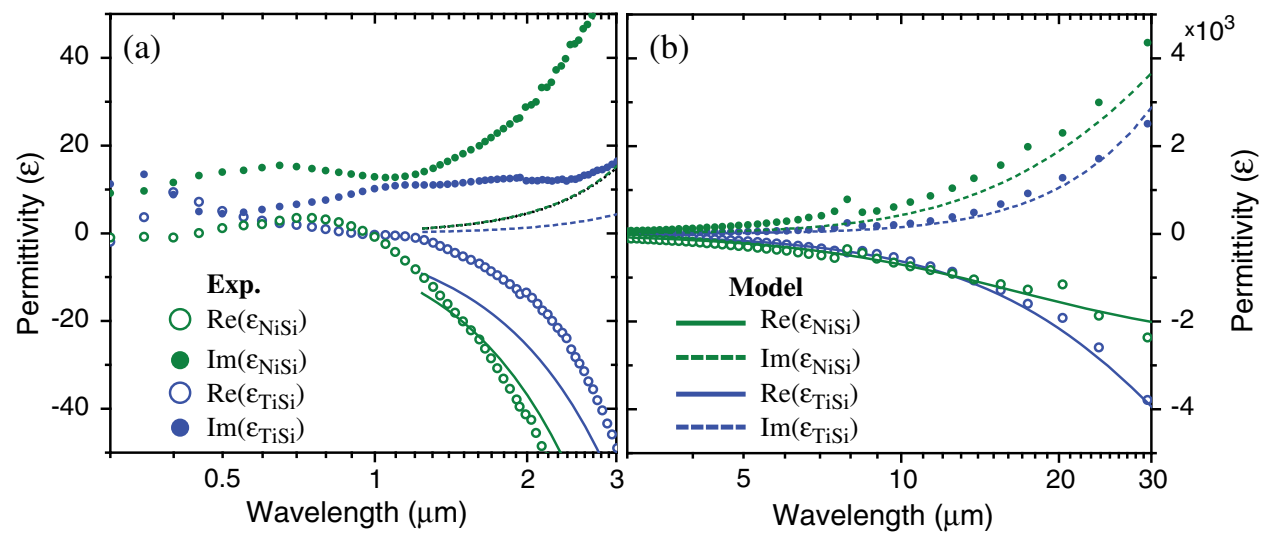

Fig. 3 Permittivity of representative silicides (NiSi-green, TiSi-blue) at (a) short $(0.3-3 \mu \mathrm{m})$ and (b) long $(3-30 \mu \mathrm{m})$ wavelengths showing both experimental data for the real (empty circle) and imaginary (filled circle) parts of the silicide permittivity. A Drude model for the real (solid) and imaginary (dashed) permittivity of both silicides is overlaid in both (a) and (b) in order to demonstrate the deviation from the free electron model at short wavelengths (a) resulting from interband absorption (experimental data and Drude model parameters from Ref. 22).

in particular, are a mature materials system, with a long history as transparent contacts for optoelectronic devices, and thus a key component in today's active display technologies. ${ }^{27}$

The aforementioned materials have plasma frequencies in the shortwave IR to UV spectral range and are thus plasmonic, or optically metallic, in the mid-IR. However, for many applications, as we will demonstrate, the ideal plasmonic material should have a magnitude for the real part of the permittivity on the order of the permittivity of the surrounding dielectric. ${ }^{28}$ Thus, for mid-IR applications, it is often desired to have plasma frequencies either in, or in close proximity to, the mid-IR. Not surprisingly, this can be achieved by further decreasing the free-carrier concentration from that of traditional plasmonic metals, the conducting oxides, or the silicides. Because of the lower free-carrier concentrations in such materials, we most often consider them "doped," as the atoms donating free electrons make up only 1 in every $10^{3}$ to $10^{7}$ atoms in the host crystal. In particular, doped semiconductors have been shown to exhibit plasmonic properties from the THz to mid-IR frequencies. ${ }^{29-32}$ The advantages of doped semiconductors as midIR plasmonic materials are many. First, these materials can be grown epitaxially, offering singlecrystal materials with high mobility and accurate doping concentration control, as well as the potential for integration with semiconductor optoelectronic structures. In addition, the doping of semiconductors can be achieved in a number of manners, including ion implantation, surface diffusion techniques, or during epitaxial growth, all of which are compatible with mature semiconductor processing and/or growth techniques. Moreover, with the correct choice of a semiconductor, one can be assured of a plasma frequency far from any interband absorption, something that cannot be said about the noble metals at shorter wavelengths. Finally, the well-known Burstein-Moss effect, ${ }^{33,34}$ where the interband absorption edge for a semiconductor blue-shifts with increasing dopant concentration (as states in the conduction or valence bands are filled, thus preventing excitation to these filled states), can extend the onset of interband absorption to energies well above the bulk semiconductor band edge. ${ }^{35}$ Figure 4 shows the real and imaginary parts of the permittivity for a variety of doped semiconductors, including MBE-grown $\mathrm{InSb}^{36}$ and InAs, ${ }^{35,37}$ and spin-doped ${ }^{38,39}$ and ion-implanted $\mathrm{Si}^{40}$

As discussed above, negative permittivity can also be achieved via the excitation of collective lattice vibrations, or phonons, in polar materials. While optical phonon energies for such materials tend to lie toward or beyond the long-wavelength end of the mid-IR, materials with high-energy phonons can show negative permittivity in the 10 to $30 \mu \mathrm{m}$ range. In Fig. 5, we show the real and imaginary permittivity for $\mathrm{SiC}\left(\lambda_{\mathrm{TO}} \sim 12.5 \mu \mathrm{m}\right),{ }^{41}$ the perovskite $\mathrm{SrTiO}_{3}\left(\lambda_{\mathrm{TO}} \sim 18 \mu \mathrm{m}\right),{ }^{42} \mathrm{GaN}\left(\lambda_{\mathrm{TO}} \sim 19 \mu \mathrm{m}\right),{ }^{43}$ and at the far end of the mid-IR spectrum, GaP $\left(\lambda_{\mathrm{TO}} \sim 28 \mu \mathrm{m}\right) .{ }^{44,45}$ The wavelength range over which each of these materials can support surface modes is much narrower than the previously discussed metals, though in this narrow range, each 

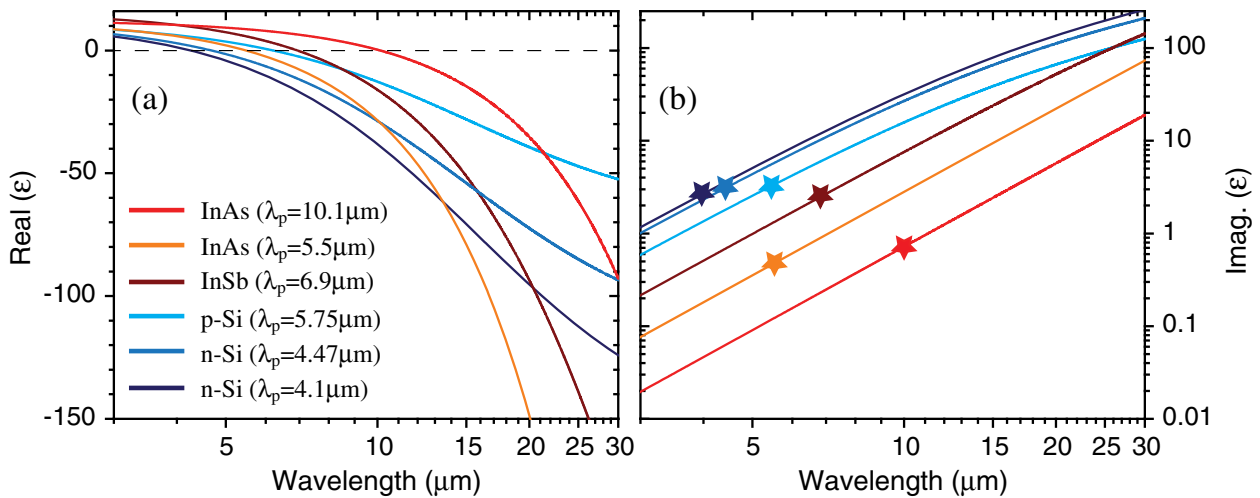

Fig. 4 (a) Real and (b) Imaginary permittivity for doped semiconductors: epitaxial grown InAs with plasma frequencies $\lambda_{p}=5.5 \mu \mathrm{m}$ (orange) ${ }^{37}$ and $10.1 \mu \mathrm{m}$ (red), ${ }^{35}$ epitaxially grown InSb with $\lambda_{p}=$ $6.9 \mu \mathrm{m}$ (wine), ${ }^{36}$ spin-doped n-type Si with $\lambda_{p}=4.1 \mu \mathrm{m}$ (navy), ${ }^{38}$ and ion-implanted n-type (blue) and p-type (cyan) with $\lambda_{p}=4.47 \mu \mathrm{m}$ and $\lambda_{p}=5.75 \mu \mathrm{m}$, respectively. ${ }^{40}$ Stars in (b) show value of imaginary permittivity at $\lambda_{p}$.

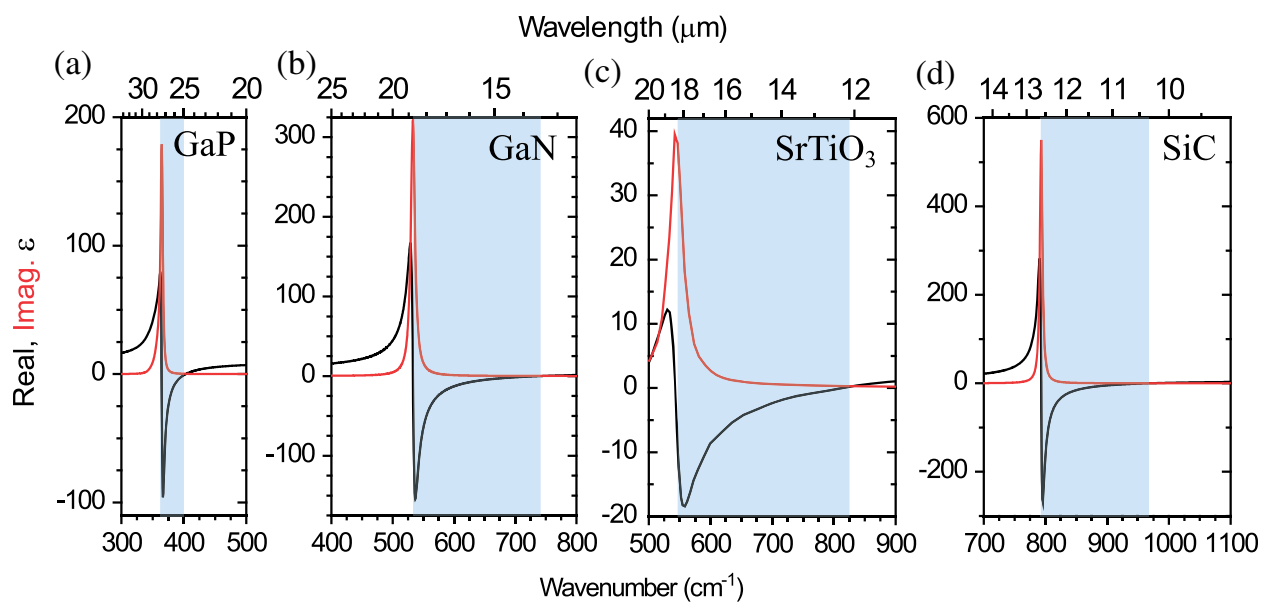

Fig. 5 Real (black) and imaginary (red) components of the permittivity for (a) GaP, ${ }^{44,45}$ (b) $\mathrm{GaN}^{43}$ (c) the perovskite $\mathrm{SrTiO}_{3},{ }^{42}$ and (d) $\mathrm{SiC}^{41}$ Shaded areas show the region of negative real permittivity for each material. All plots share a common scale (in energy, or wavenumber) to compare spectral range over which real permittivity is negative.

of the above phononic metals, macroscopically, will exhibit similar optical properties to tradition plasmonic metals (high reflectivity, small skin depths, etc.).

Finally, we will discuss graphene, though separately from all of the above materials. Graphene is a material of significant current research interest with a host of unique and appealing properties. At the same time, the physics of surface plasmons on graphene is significantly different than that for metals, in particular, because we can define traditional plasmonic materials with a permittivity, which represents, in some sense, the volumetric polarization of the media. Only one atomic layer thick, graphene cannot be modeled with a volumetric permittivity, and for this reason, we consider graphene separately from our other mid-IR plasmonic materials (in Sec. 5).

\section{Material Comparisons for Plasmonic and Epsilon-Near-Zero Applications}

Within the larger field of plasmonics, we can divide plasmonic structures into three primary (and reasonably differentiated) categories. In the first, we place plasmonic structures supporting localized surface plasmon (LSP) excitations. The second would consist of structures supporting 
propagating, surface plasmon polariton (SPP) modes, and the final, somewhat more amorphous category, would consist of plasmonic structures acting as constituent subwavelength inclusions in a larger metamaterial or metasurface materials. The broad range of the proposed and demonstrated metamaterial and surface structures makes the development of a single metric by which to measure the suitability of a plasmonic material for such applications unrealistic. Moreover, because this paper is focused on plasmonic materials and not metamaterials (a large and varied field in and of itself), we leave such analysis for future discussion. Adjacent to the field of plasmonics, though not unrelated, is the study of epsilon-near-zero (ENZ) materials, or materials, as the name suggests, having vanishingly small dielectric permittivity. It is here, adjacent to our materials' spectral range of plasmonic behavior, that we begin our comparison of the plasmonic materials under discussion. As our discussion continues, we will move from regions of near-zero permittivity (ENZ), to regions of small, negative real permittivity (LSPs) and finally to plasmonic modes supported in regions of increasingly negative real permittivity (SPPs).

\subsection{ENZ Materials}

For the plasmonic and phononic materials discussed above, ENZ permittivity will occur at frequencies near the transition from positive to negative permittivity, in other words, for Drude metals, near the plasma frequency $\left(\omega_{p}\right)$, and for phononic materials, near the longitudinal optical phonon energy $\left(\omega_{\mathrm{LO}}\right)$. An electromagnetic wave propagating within an ENZ material will exhibit a dramatic increase in local wavelength (as the refractive index becomes vanishingly small). Such materials have been of significant recent interest, resulting in large part from simulation and theory, as well as microwave frequency experimental, results suggesting that ENZ materials can allow for near perfect coupling between waveguiding structures, ${ }^{46,47}$ or control of radiating phase patterns ${ }^{48}$ among other novel effects. ${ }^{49}$ The majority of ENZ experimental demonstrations have been performed at microwave frequencies, where composite materials or waveguiding structures can be designed to mimic bulk ENZ behavior. At optical frequencies, many plasmonic or phononic materials offer the opportunity to serve as bulk ENZ materials (albeit across a narrow frequency range). However, it should be cautioned that not every plasmonic material will make for a suitable bulk ENZ material at the plasma frequency. In particular, metals with strong interband absorption near the plasma frequency, such as the noble metals and the intermetallics (as can be seen in Figs. 2 and 3), will have large imaginary components of permittivity, resulting in unacceptable losses at ENZ frequencies. Furthermore, "designer" plasmonic metals, such as the doped oxides and intermetallic silicides, have only been demonstrated with plasma frequencies in the near-IR, and thus are not considered in our comparison.

Doped semiconductors and phononic materials, on the other hand, have plasma frequencies well below their band-edges (with only one known exception), ${ }^{36}$ and thus can serve as relatively low-loss, mid-IR bulk ENZ materials. As one such example, the use of heavily doped semiconductors as ENZ materials was demonstrated to enhance the transmission of light through subwavelength apertures. ${ }^{50,51}$ For any bulk ENZ material, we can describe wave propagation with a complex wavevector, $k=\omega \sqrt{\varepsilon \mu}$, which effectively determines the propagation length and local wavelength of light at ENZ frequencies. Assuming $\mu=\mu_{o}$ (as we will throughout this paper), we can gain an effective measure of the quality of any bulk ENZ material by determining the imaginary component $(\kappa)$ of the complex refractive index $(\tilde{n}=n+i \kappa)$ at the wavelength $\lambda_{\mathrm{ENZ}}$, where the real part of the refractive index is minimum $\left[n\left(\sim \lambda_{\mathrm{ENZ}}\right)\right]$. Figure 6(a) plots both $n\left(\sim \lambda_{\mathrm{ENZ}}\right)$ (red) and $\kappa\left(\sim \lambda_{\mathrm{ENZ}}\right)$ (blue) for a variety of mid-IR plasmonic and phononic materials, whereas Fig. 6(b) plots the normalized (to free-space wavelength) local wavelength and penetration depth $\left(I\left(\delta_{I}\right)=I_{o} e^{-2}\right)$ for the materials in Fig. 6(a). The low losses of the phononic materials at $\sim \lambda_{\mathrm{ENZ}}$ suggest that such materials are ideal for ENZ applications, however, the inflexibility of the ENZ wavelength in such material systems is a significant limiting factor. Doped semiconductors, on the other hand, while having larger losses, allow for control of the ENZ wavelength, making such materials attractive for ENZ applications across a large portion of the mid-IR. 

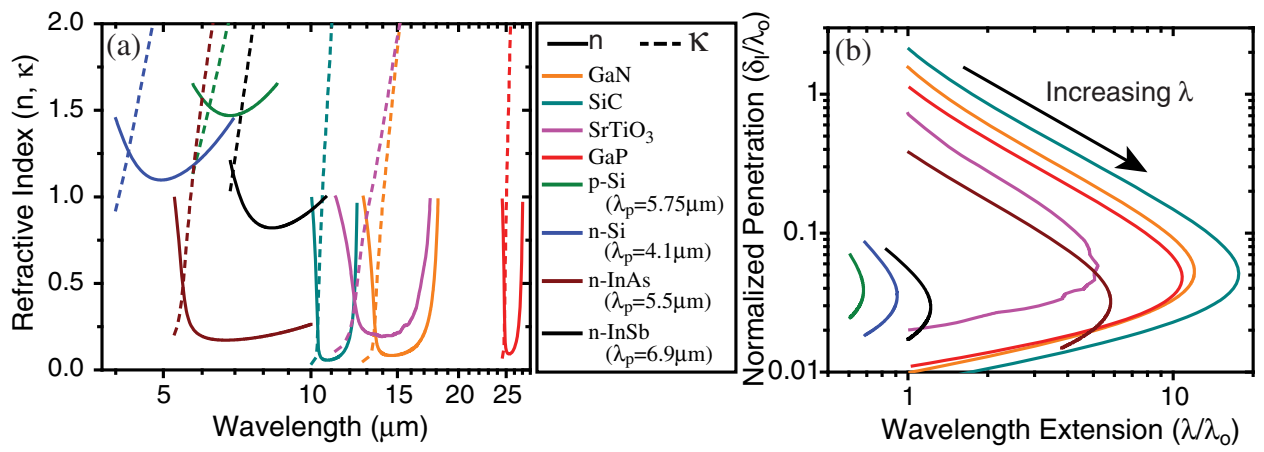

Fig. 6 Comparison of mid-IR materials for epsilon-near-zero (ENZ) applications. (a) Real (solid) and imaginary (dashed) components of complex refractive indices for doped semiconductors and phononic materials vs. wavelength. (b) Normalized wavelength and penetration depth (for intensity) for the mid-IR phononic and plasmonic materials in (a).

\subsection{Localized Surface Plasmons}

The LSP is a hybrid excitation of free-charge carriers and an electromagnetic mode tied to the metal/dielectric interface of a 3-D metallic structure. The strong interaction of light with subwavelength metallic particles supporting LSPs has been used for centuries, for instance, in stained-glass windows ${ }^{52}$ though it is only recently that advances in nanofabrication have allowed for controllable and repeatable fabrication of nanoscale metallic structures using solution-based approaches ${ }^{53}$ or alternatively, via nanolithography, for integration with chip-scale optical or optoelectronic devices and structures. The standard analysis for an LSP assumes a subwavelength metallic sphere of diameter $a$, with $\lambda_{o} \gg a$, modeled using the quasistatic approximation, which assumes a uniform incident electric field across the entire metallic particle. Simple electrostatics can then be used to solve for the electric field both inside and outside of the sphere. Outside the sphere, the incident electric field $\left(E_{o}\right)$ is supplemented by the field resulting from the polarization of the metallic nanoparticle, which is resonant, with an amplitude given by

$$
E_{\mathrm{LSP}} \propto\left[\frac{\varepsilon_{m}-\varepsilon_{d}}{\varepsilon_{m}+\chi \varepsilon_{d}}\right] a^{3} E_{o} .
$$

Here, $\varepsilon_{m}$ and $\varepsilon_{d}$ are the frequency-dependent permittivity of the metallic nanosphere and the surrounding dielectric, respectively, and $\chi$ is a geometric factor ( 2 for a perfect sphere, but potentially larger for structures with larger aspect ratios).$^{54,55}$ Thus, for a perfect, ultra-subwavelength sphere in air, the LSP resonance will occur when the permittivity of the nanosphere material is $\varepsilon_{m}=-2$. For most traditional plasmonic materials (i.e., $\mathrm{Au}, \mathrm{Ag}$ ), this resonance occurs at visible frequencies. In order to push this resonance to longer wavelengths, the index of the surrounding dielectric can be increased, though realistically the choice of surrounding dielectric is often constrained by the application under consideration. Alternatively, changing the size and/or shape of the plasmonic particle can also result in significant red-shifts. However, it should be noted that continued "stretching" of the plasmonic particle, while continuing to red-shift the resonant wavelength, will also move the LSP resonance into a wavelength range where most metals are nearperfect conductors $\left(\left|\varepsilon_{m}\right| \gg 1\right)$, which eventually results in a structure whose optical properties more closely resemble a traditional antenna than a subwavelength plasmonic resonator. ${ }^{56}$ Thus, while impressive results have been obtained using noble-metal nano/microantennas, ${ }^{57-59}$ true subwavelength resonant devices can only be achieved when the permittivity of the metal is of the same order of magnitude as the surrounding dielectric $\left(\left|\varepsilon_{m}\right| \sim \varepsilon_{d}\right)$. Here, the "designer" metals of the mid-IR and the phononic materials with mid-IR frequency phonon resonances offer opportunities for engineering LSP resonances not available with traditional plasmonic metals ${ }^{60,61}$ A direct comparison of materials for LSP applications, however, might seem non-trivial, given the wide variety of sizes and geometries (shells, ${ }^{5,62}$ cubes, ${ }^{63}$ crosses, ${ }^{64}$ rods,${ }^{55}$ spheres, ${ }^{65}$ nanoprisms ${ }^{66}$ etc.) used to support such resonances. 
Below, we use two approaches to compare materials for LSP applications. In Fig. 7(a), we plot the absorption enhancement $\left(Q_{\text {abs }}\right)$ for mid-IR phononic and plasmonic materials. This absorption enhancement is directly related to the electric field enhancement in the vicinity of a perfect plasmonic sphere in vacuum, with a scaled radius $r_{o}=\lambda_{o} / 10$, using the expression from Ref. 67:

$$
Q_{\mathrm{abs}}=\frac{24 \pi r}{\lambda} \frac{\varepsilon_{d}^{3 / 2} \varepsilon_{m}^{\prime \prime}}{\varepsilon_{m}^{\prime 2}+\left(\varepsilon_{m}^{\prime}+2 \varepsilon_{d}+48 \pi^{2} r^{2} \varepsilon_{d}^{2} / 5 \lambda^{2}\right)^{2}}
$$

Alternatively, we can use the quality factor $\left(Q_{\mathrm{LSP}}\right)$ of a plasmonic nanostructure as our FOM [shown in Fig. 7(b)], which gives the spectral sharpness of the LSP response, and depends only on the plasmonic material and not the size and shape of the nanoparticle or even the surrounding dielectric (assuming a lossless, dispersionless medium) ${ }^{68,69}$ For such a system, we can write the $Q$ of the nanoplasmonic structures in the quasistatic ${ }^{70}$ limit as

$$
Q_{\mathrm{LSP}}=\omega \frac{\mathrm{d} \varepsilon_{m}^{\prime}}{\mathrm{d} \omega} / 2 \varepsilon_{m}^{\prime \prime} .
$$

The LSP quality factor $\left(Q_{\mathrm{LSP}}\right)$ of a plasmonic material provides a more general, geometryindependent, indication as to the suitability of plasmonic material for LSP applications, while the absorption enhancement $\left(Q_{\text {abs }}\right)$, which is directly related to the LSP extinction coefficient and field enhancement for a set geometry, allows for comparison of materials with identical geometries and surrounding dielectrics.

As mentioned above, a true LSP resonance occurs in the spectral range where $\varepsilon_{m}^{\prime}$ is on the order of the permittivity of the surrounding dielectric. For this reason, we confine our plotted $Q$ 's in Fig. 7 to the range where $-2>\varepsilon_{m}^{\prime}>-20$ and exclude the material systems discussed above (noble metals, transparent conducting oxides, silicides, etc.) which have near-IR or visible plasma frequencies, and thus large, negative permittivity in the $3<\lambda_{o}<30 \mu \mathrm{m}$ range. As can be seen in Fig. 7, both of the plots indicate that the considered phononic materials have the highest FOMs for LSP applications, though again, across very limited wavelength ranges. Nonetheless, the semiconductors with the lowest losses give FOMs comparable to (or greater than) noble and alkali metals, ${ }^{67,69}$ conducting oxides and transition metal nitrides, ${ }^{71}$ and the silicides. ${ }^{22}$

\subsection{Propagating Surface Plasmons}

The fundamental building block of the propagating surface plasmon is the single-interface SPP, a hybrid mode propagating at the interface between a conducting plasmonic material and an overlying dielectric/insulator (MI). Propagating plasmonic modes, however, can be supported by a variety of (usually planar) structures consisting of alternating metal and dielectric layers.
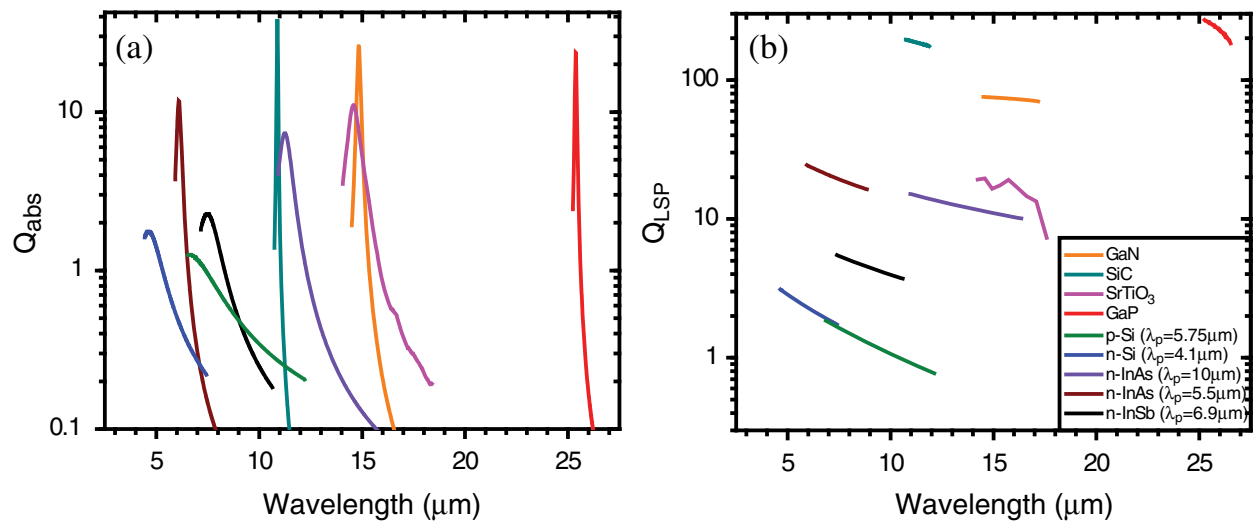

Fig. 7 (a) Calculated absorption efficiency $\left(Q_{a b s}\right)$ and (b) quality factor $\left(Q_{\mathrm{LSP}}\right)$ for selected mid-IR plasmonic and phononic materials. 
Of these, the most commonly studied are the metal-insulator-metal (MIM) and insulator-metalinsulator (IMI) waveguides. The basic geometry of all three of the aforementioned plasmonic waveguides, along with sketched field intensities of the supported plasmonic modes, are shown in Figs. 8(a), 8(c), and 8(d). The IMI structure can be thought of, essentially, as two SPP modes coupled across a thin metal layer. These structures support both symmetric and antisymmetric modes and have been demonstrated to allow for long propagation lengths for the coupled surface waves, especially in the antisymmetric configuration which is often referred to as a long-range surface plasmon polariton (LRSPP). ${ }^{72}$ The MIM structure allows for strong localization of the plasmonic mode, via the additional confinement provided by the upper plasmonic surface. These structures are of significant interest for applications where strong light-matter interaction is desired and have been used as waveguides for plasmonic lasers ${ }^{73}$ as well as for switchable waveguiding structures. ${ }^{74}$ While the MI, MIM, and IMI serve as the most fundamental SPP configurations, and those most easily characterized analytically, a wide variety of structures supporting propagating plasmonic modes have been investigated, including dielectric-loaded MI laser waveguides, ${ }^{75}$ slot waveguides, ${ }^{76-79}$ toothed, or modulated, MIM waveguides, ${ }^{80}$ multilayered dielectric or metal waveguides, and even linear arrays of localized plasmonic resonators, capable of supporting propagating waves. ${ }^{81}$ Below, we constrain our analysis to the most fundamental MI waveguides, with a brief discussion of the MIM and IMI, operating with the not unreasonable assumption that the materials best suited for these waveguide structures will also serve well for more complicated systems.

The single-interface SPP mode can be easily obtained by solving Maxwell's equations at a metal/dielectric interface, assuming a propagation vector along the interface, and evanescent decaying field intensities into both the metal and dielectric half-spaces. The resulting dispersion relation for the SPP wavevector is well-known and commonly expressed as

$$
k_{\mathrm{SPP}}=\frac{\omega}{c} \sqrt{\frac{\varepsilon_{m} \varepsilon_{d}}{\varepsilon_{m}+\varepsilon_{d}}} \quad \varepsilon_{m}<0, \quad\left|\varepsilon_{m}\right|>\left|\varepsilon_{d}\right| \text {, }
$$

where $\varepsilon_{m}$ and $\varepsilon_{d}$ are the (complex) relative permittivity of the plasmonic metal and the overlying dielectric, respectively. In order to support such a mode, the permittivity of the metal must be of the opposite sign and larger in magnitude than the dielectric material's permittivity. The plot in Fig. 8(b) shows the normalized dispersion curve for a single-interface Drude metal/air SPP waveguide for varying scattering rates $\gamma$ [Eq. (1)]. From this, we can first see that $k_{\mathrm{SPP}}$, at a given frequency, is always larger than the magnitude of the incident light wavevector, meaning that for even grazing incidence angles, a momentum mismatch exists between incident light and the SPP. This mismatch is generally overcome either by prism coupling (the Kretchman or Otto configurations) or alternatively, via grating coupling. In addition, from the dispersion curve, we see a dramatic extension of the SPP wavevector (and thus an equivalent compression of the SPP wavelength) that occurs only near the surface plasmon frequency, defined as $\omega_{s p}=\omega_{p} / \sqrt{1+\varepsilon_{d}}$. However, at frequencies near $\omega_{s p}$, we also observe a significant increase in the imaginary component of $k_{\mathrm{SPP}}$, indicating significant losses for the propagating mode. This

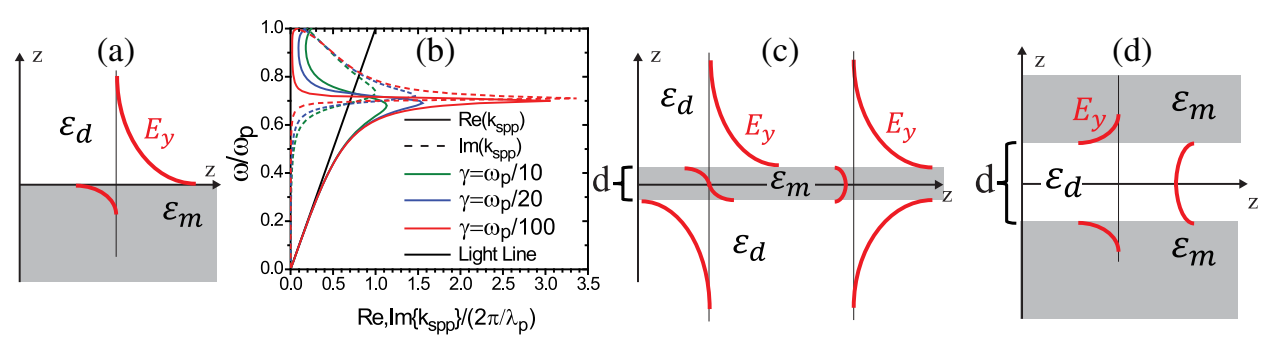

Fig. 8 Schematics of the three fundamental structures supporting propagating plasmonic modes. (a) Single interface metal-insulator (MI), (c) dual interface insulator-metal-insulator (IMI) and (d) dual interface metal-insulator-metal (MIM) waveguides. (b) Dispersion relation showing real (solid) and imaginary (dashed) components of $k_{\mathrm{SPP}}$ for Drude metal/air interface with varying metal losses $\gamma=\omega_{p} / 10$ (green), $\omega_{p} / 20$ (blue), $\omega_{p} / 100$ (red). Solid black line is the light line. 
points to the inherent trade-off associated with all three SPP modes: significant localization of the electromagnetic mode is possible (with subwavelength confinement achievable for frequencies near $\omega_{s p}$ ), but at the expense of the mode's propagation length.

The SPP propagation length can be defined as the length at which the electric field or the intensity of the SPP mode decays to $1 / e$ of its original value. Here, we use the expression for the decay of the SPP field strength, which can be expressed as the inverse of the imaginary part of the SPP wavevector as ${ }^{82}$

$$
L_{\mathrm{SPP}}=\frac{1}{\operatorname{Im}\left(k_{\mathrm{SPP}}\right)}
$$

The lateral component of the SPP wavevector can be determined from the expressions $\varepsilon_{i} k_{o}^{2}=k_{\mathrm{SPP}}^{2}+k_{y, i}^{2}$, where $k_{o}=\omega / c$, and the $i$ subscripts indicate either the dielectric $(d)$ or metal $(m)$ media. For purposes of comparison, we will assume the dielectric media to be air, with $\varepsilon_{d}=1$. Because $k_{\mathrm{SPP}}>k_{o}=\omega / c, k_{y, i}^{2}$ will always be negative, and thus the transverse component of the SPP mode wavevector will decay into both the metal and dielectric. We can then write the metal $\left(\delta_{m}\right)$ and dielectric $\left(\delta_{d}\right)$ penetration depths as

$$
\delta_{m}=1 / \operatorname{Re}\left\{k_{o} \sqrt{\frac{-\varepsilon_{m}^{2}}{1+\varepsilon_{m}}}\right\}, \quad \delta_{d}=1 / \operatorname{Re}\left\{k_{o} \sqrt{\frac{-1}{1+\varepsilon_{m}}}\right\} .
$$

For frequencies far from the surface plasmon frequency $\left(\omega_{s p}\right)$, the SPP mode confinement is defined entirely by the penetration into the dielectric (since $\left|\varepsilon_{m}\right| \ll \varepsilon_{d}$, and thus the field in the metal is weak and decays rapidly). Near the surface plasmon frequency, however, penetration of the mode into the metal must be considered, which leads to the expression below for the SPP confinement: ${ }^{83-85}$

$$
\delta_{\mathrm{SPP}}=\left\{\begin{array}{ll}
\delta_{d}, & \left|\varepsilon_{m}\right| \geq e \varepsilon_{d} \\
\delta_{d}+\delta_{m} \ln \frac{e \varepsilon_{d}}{\left|\varepsilon_{m}\right|}, & \left|\varepsilon_{m}\right|<e \varepsilon_{d}
\end{array} .\right.
$$

From the above expressions, we can plot both the confinement and penetration depth of a mid-IR SPP mode for the materials investigated in this work. Unlike the case of the LSP, which we argued can only be sustained by plasmonic/phononic materials with a permittivity of the same order of magnitude as the surrounding dielectric (thus excluding the noble metals, transparent conducting oxides and nitrides, and the silicides), all of the materials discussed in this work will be able to sustain SPPs across some portion of the mid-IR. We thus plot only representative materials in Fig. 9, where we show the wavelength-dependent SPP propagation length $\left(L_{\mathrm{SPP}}\right)$, mode width $\left(\delta_{\mathrm{SPP}}\right)$, and FOMs $M_{1 \mathrm{SPP}}=L_{\mathrm{SPP}} / \delta_{\mathrm{SPP}}$ and $M_{2 \mathrm{SPP}}=\varepsilon_{m}^{\prime \prime 2} / \varepsilon_{m}^{\prime}$, for the phononic materials $\left(\mathrm{GaN}, \mathrm{SrTiO}_{3}, \mathrm{SiC}, \mathrm{GaP}\right), n$-doped semiconductors $\operatorname{InAs}\left(\lambda_{p}=5.5 \mu \mathrm{m}\right)$ and Si $\left(\lambda_{p}=4.1 \mu \mathrm{m}\right)$, representative transparent conducting oxides and nitrides (AZO, TiN), the noble metal $\mathrm{Au}$, and the silicide TiSi. The FOM $M_{1 \mathrm{SPP}}$ provides a clear illustration of the confinement/propagation length trade-off, ${ }^{86}$ while $M_{2 \mathrm{SPP}}$ is the simpler metric for determining a material's suitability for SPP-based applications, directly extracted from material permittivity. ${ }^{71}$

As can be seen in Fig. 9, noble metals show the largest FOM for SPPs at mid-IR wavelengths, due in large part to their extremely large, negative permittivity in the mid-IR (far from their plasma frequency). However, the noble metal plasmonic mode extends well over $10 \mu \mathrm{m}$ into the dielectric material across the entire frequency range (and up to $\sim 1000 \mu \mathrm{m}$ at the long wavelength edge of the mid-IR), making noble metal-based SPP structures entirely unsuitable for sub-wavelength optics at mid-IR wavelengths. For mode confinement below a free-space wavelength, only doped semiconductors and phononic materials can provide the required confinement. Interestingly, the silicides (illustrated in Fig. 9 by TiSi) provide a reasonable compromise between long propagation length and high FOM, and reasonable confinement factors (multiple wavelengths). Combined with their Si-processing compatibility, the silicides live up to their billing as potential plasmonic waveguiding materials at IR frequencies.

The IMI and MIM SPPs are more complicated to characterize than their single interface MI counterpart. In addition to requiring the solution of transcendental equations in a complex wave 

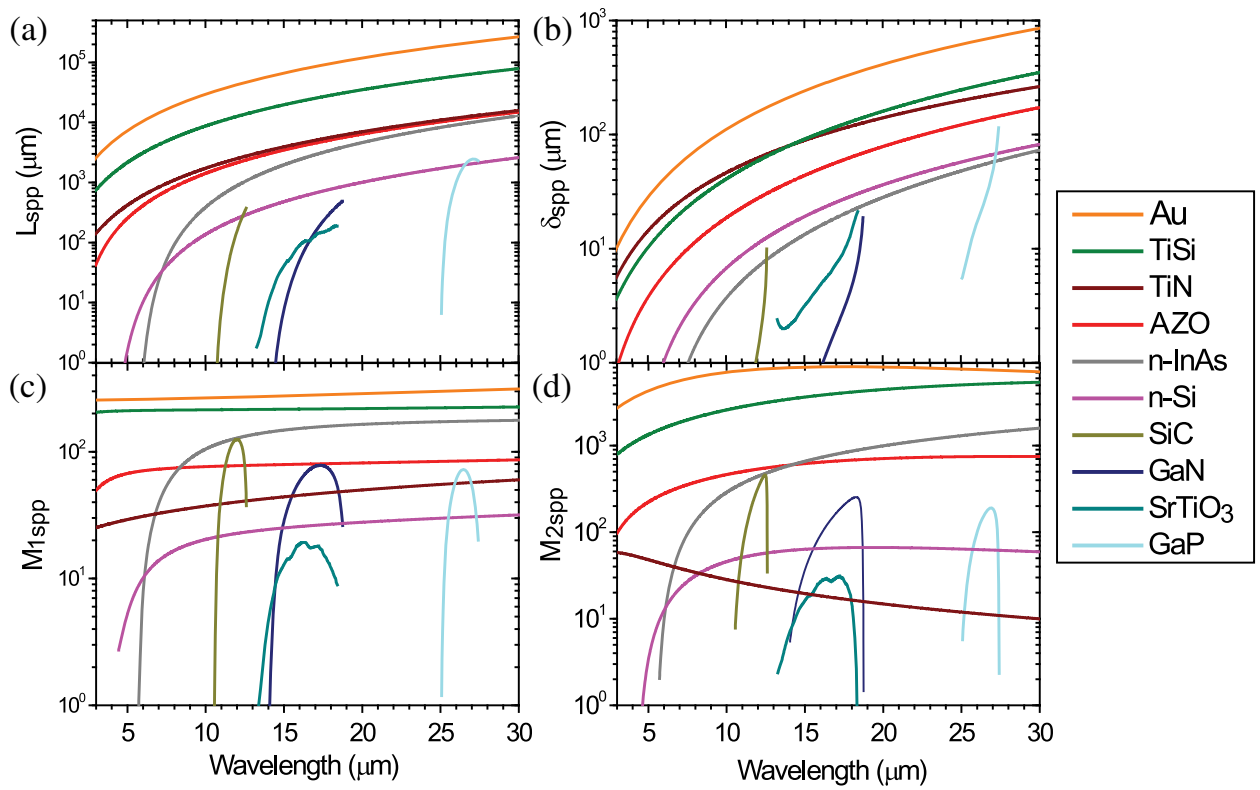

Fig. 9 SPP characteristics for a variety of plasmonic matetrials. (a) Propagation length, (b) mode confinement, figures of merit (c) $M_{1 \mathrm{SPP}}=L_{\mathrm{SPP}} / \delta_{\mathrm{SPP}}$ and (d) $M_{2 \mathrm{SPP}}=\varepsilon_{m}^{\prime \prime 2} / \varepsilon_{m}^{\prime}$, as a function wavelength.

vector space, each of these modes has an additional parameter space to be explored (for the IMI, it is the metal thickness, for the MIM, the air spacer thickness). The additional parameters make comparison across materials and wavelengths exceedingly difficult. Despite the greater complexity associated with these modes, they have been studied extensively as each have features which lend themselves to a number of potential applications. A MIM structure can support propagating modes down to ultra-subwavelength dimensions, which has potential applications for on-chip interconnects or alternatively, as subwavelength cavities for plasmonic lasers. ${ }^{73}$ IMI structures, as shown in Fig. 8(c), can support both field antisymmetric (left) and symmetric (right) modes. As the metal thickness decreases, the attenuation of the symmetric mode decreases, and this mode begins to more closely resemble a plane wave. The combination of low attenuation (orders of magnitude smaller than the single interface SPP) and a plane wave-like mode profile (which simplifies coupling to free-space radiation) makes the thin metal IMI symmetric mode (the LRSPP) ideal for a variety of waveguiding applications.

While exact calculations of attenuation and mode confinement for IMI and MIM modes require numerical approaches to obtain the complex SPP wavevector $k_{\mathrm{SPP}}$, the basic results found for the single-interface SPP will describe the relative quality of mid-IR plasmonic materials for IMI and MIM SPP waveguides. This approach can be extended to more complicated, 2-D and 3-D, SPP structures such as ribbon or slot waveguides, nanowire, or even plasmonic cavities. $^{87-93}$

\section{Graphene}

Graphene has been recently investigated as an alternative long wavelength plasmonic material because of its unique band structure and very high mobility. ${ }^{94-96}$ Graphene is a single layer of carbon atoms tightly packed in a honeycomb lattice. Each carbon atom is sp2 hybridized and leaves $2 p$ out-of-plane orbitals to form $\pi$-bonds extending over the surface. The 2-D nature of graphene is not necessarily unique among plasmonic materials. It has been known for some time that 2-D electron gases in semiconductor materials can support plasmonic excitations at $\mathrm{THz}$ frequencies, ${ }^{97}$ with gate-tuning of the $2 \mathrm{DEG}$ able to control carrier concentration, and thus the plasmon resonance. ${ }^{98}$ In some sense, efforts in graphene plasmonics are an exciting extension of this early work, providing a room temperature 2-D material with the potential for high carrier concentrations and thus plasmonic behavior in the mid-IR. ${ }^{99-101}$ Graphene's unique band structure includes a linear energy dispersion relation with a vanishing electronic density of states near 
the Fermi level $\left(E_{\mathrm{F}}\right)$ and zero energy band gap at the Dirac point [Fig. 10(b)]. The Fermi-level of graphene is given as $E_{\mathrm{F}}=\hbar v_{\mathrm{F}} k_{\mathrm{F}}$, where $\hbar$ is Plank's constant, $v_{\mathrm{F}} \sim 10^{6} \mathrm{~m} / \mathrm{s}$ is the Fermi velocity, and $k_{\mathrm{F}}=\sqrt{\pi n}$ is the Fermi wave vector, with $n$ as the free-carrier concentration, which can be controlled either by application of a gate bias ${ }^{102,103}$ or via chemical doping. ${ }^{104-106}$ Accordingly, graphene's 2-D plasma resonance scales as $n^{1 / 4}$. Adding carriers to graphene opens up a band gap $\left(E_{\mathrm{g}}=2 E_{\mathrm{F}}\right)$, and with carrier concentrations as high as $n=3-7 \times 10^{13} \mathrm{~cm}^{-2}$ achieved, the resulting bandgap $E_{\mathrm{g}} \sim 0.64-1 \mathrm{eV}$ allows for low-loss plasmonic modes in the $\mathrm{THz}$ and well into the mid-IR. ${ }^{107,108}$ At higher energies $\left(\hbar \omega>2 E_{\mathrm{F}}\right)$, the real part of the optical conductivity will reach a universal value, as shown in Fig. 10(a), resulting from interband transitions, and effectively dampen any potential plasmonic modes. ${ }^{109-111}$

Graphene, and other 2-D materials supporting plasmonic modes, cannot be modeled using the volumetric relative permittivity we have used to model the other plasmonic materials studied in this work. Instead, the graphene layer is most often treated as a thin conducting sheet with a complex frequency-dependent optical conductivity. Though, to date, limits on carrier concentration have prevented the extension of graphene plasmonics into the near-IR and VIS wavelength ranges, this novel material provides the potential for a low-loss and tunable plasmonic material well-suited for mid-IR applications. For energies well below the Fermi level, the optical conductivity, $\sigma_{\mathrm{g}}(\omega)$, can be expressed using the Drude model (ignoring contributions from interband absorption), from which we can obtain the graphene plasmon dispersion, $k_{\mathrm{gsp}}(\omega)$

$$
\sigma_{\mathrm{g}}(\omega)=\frac{e^{2} E_{F}}{\pi \hbar^{2}} \frac{1}{\omega+i \gamma}, \quad k_{\mathrm{gsp}}(\omega) \approx\left(\frac{\hbar^{2}}{4 e^{2} E_{\mathrm{F}}}\right)\left(\varepsilon_{d}+1\right) \omega(\omega+i \gamma),
$$

where $\varepsilon_{d}$ is the permittivity of the surrounding dielectric, assuming a symmetric dielectric/graphene/dielectric structure. Figure 10 shows both the calculated Fermi level-dependent conductivity of graphene as well as the dispersion relation for plasmons on a graphene sheet surrounded by air (with $E_{\mathrm{F}}=0.5 \mathrm{eV}$ ). For comparison, we also show the dispersion relation for $n$-doped $\operatorname{InAs}\left(\lambda_{p}=5.5 \mu \mathrm{m}\right)$ and $\mathrm{Au}$ (modeled as a Drude metal). As can be clearly seen from the figure, when compared to $\mathrm{Au}$, and even to doped InAs, graphene plasmons in the mid-IR show a dramatic wavelength compression $\left(k_{\|}\right.$large), indicating strong confinement of the propagating mode. Such a strong confinement in the atomically thin graphene layer is remarkable but also points to a significant challenge associated with graphene plasmonics, namely the difficulty in coupling free-space radiation to these modes. For this reason, many of the clearest studies of propagating plasmons on graphene have utilized unique near-field scanning optical microscopes operating at mid-IR frequencies, which allow near-field coupling of free-space radiation to graphene plasmons via a nanoscale scattering tip. ${ }^{112,113}$ Alternatively, nanoscale graphene structures, such as ribbons or disks, can allow for coupling into localized plasmonic modes, and such structures have been proposed and initially demonstrated for $\mathrm{THz}$ and mid-IR frequencies. ${ }^{99,100,114-116}$ The low-loss and strongly bound graphene plasmon has significant potential
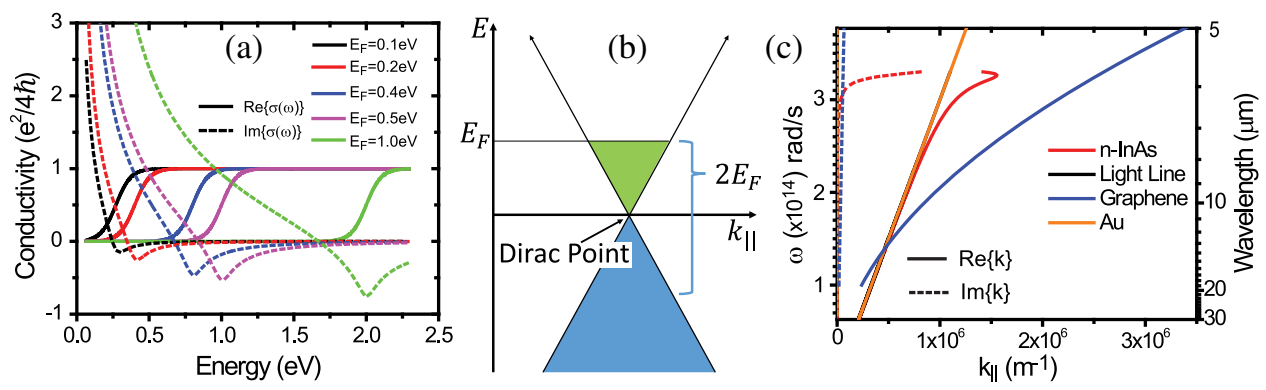

Fig. 10 (a) Real (solid) and imaginary (dashed) components of the optical conductivity of graphene calculated for different Fermi energy levels $\left(E_{\mathrm{F}}=0.1,0.2,0.5,0.5\right.$, and $1.0 \mathrm{eV}$ ). (b) Band structure and $E_{\mathrm{F}}$ for doped/electrically gated graphene. (c) Dispersion relations showing real (solid) and imaginary (dashed) propagation vectors for SPPs on graphene with $E_{F}=0.5$ (blue), InAs (red) and Au (orange). Because of the large negative permittivity of $A u$ at these long wavelengths, the Au dispersion curve overlays the light line, with the imaginary component of the Au wavevector overlaying the $y$-axis of the plot. 
for mid-IR applications, in particular benefiting from extremely strongly enhanced fields. But perhaps as appealing as the characteristics of the graphene plasmon is the potential for developing tunable plasmonic structures in the IR. The significant tuning of the graphene optical conductivity by electrostatic gating allows for the design and demonstration of broadly tunable active mid-IR plasmonic devices. ${ }^{117-120}$ While such tuning has been demonstrated experimentally and shows tremendous promise, the field of graphene plasmonics has much room to grow and is expected to constitute a significant fraction of the larger field of mid-IR plasmonics moving forward.

\section{Outlook}

For any plasmonic structure, the desired application plays a key role in determining the choice of material. The mid-IR provides a unique set of potential applications, in many ways distinct from plasmonic applications at shorter wavelengths. The abundance of vibrational and rotation absorption lines makes the mid-IR an attractive wavelength for sensing applications, which could benefit from the strong mode confinement, and thus strong light-matter interaction, provided by plasmonic materials near their plasma wavelengths. For such applications, particularly those using LSPs, materials with ENZ near or in the mid-IR are required (doped semiconductors and phononic materials), ${ }^{61}$ though impressive results have been achieved with wavelength-scale antenna structures using traditional plasmonic materials. ${ }^{57,58}$ The mid-IR is also home to peak blackbody emission wavelengths for objects with temperatures up to $\sim 1400 \mathrm{~K}$. Plasmonic materials with designed absorption resonances in the mid-IR, upon heating, can serve as spectrally selective thermal light sources, with applications for IR sensors, signals, and potentially thermophotovoltaics. For such applications, the ability to withstand high temperatures is essential, and the use of semiconductors and noble metals is unrealistic. Here the transition metal nitrides may play an increasingly important role, as they are low-cost and capable of withstanding extreme temperatures. Alternatively, $\mathrm{SiC}$ phononic resonances have been used to generate narrow bandwidth directional emission, ${ }^{121}$ though as with all of the phononic materials, the wavelength flexibility of such devices is limited. For waveguiding applications, particularly for on-chip interconnects, CMOS compatibility is essential, ruling out Au and Ag, but opening the door for the transparent conducting oxides and nitrides, the silicides, and potentially even doped Si.

Mid-IR optoelectronic components are a field of growing technological importance, with a wide variety of sensing, security, and even communication applications. Recently, the quantum cascade laser (QCL) $)^{122,123}$ and the interband cascade laser, ${ }^{124,125}$ wavelength-flexible, compact, and semiconductor-based light sources have emerged as commercially viable sources of coherent light across the mid-IR. At the same time, the renewed interest in mid-IR photonics, driven in large part by the QCL, is motivating efforts to develop improved and novel semiconductor-based IR photodetectors. ${ }^{126-129}$ The interaction of light with mid-IR optical and optoelectronic systems is often multiscale, and controlling and understanding light-matter interaction across this broad range of scales can pose a significantly more daunting challenge than it does at shorter wavelengths. The electronic transitions engineered to emit or absorb mid-IR light, or molecules to be sensed with mid-IR light, are all nanoscale in size. Yet the emitters, detectors, or waveguiding components of mid-IR optoelectronics are usually wavelength-scale or larger, while the free-space optics used for mid-IR optical systems are larger still. The ability of plasmonic structures to confine light to subwavelength dimensions opens the door to a new class of mid-IR optoelectronic devices with improved functionality provided by enhanced, or designable, light-matter interaction enabled by plasmonic structures. ${ }^{130-138}$ While noble metals have been the plasmonic material of choice for many of these applications due to low losses at mid-IR wavelengths, such materials are non-ideal for subwavelength confinement of plasmonic/optical modes, limiting the desired gains from enhanced light-matter interaction. For this reason, there is a growing interest for mid-IR applications in materials with longer plasma wavelengths. For III-V optoelectronics, this would suggest the use of III-V doped semiconductors, which can be integrated directly into optoelectronic structures during epitaxial growth, and allow for device structures, ${ }^{139}$ and even tuning schemes, ${ }^{140}$ compatible with existing III-V optoelectronic architectures. 


\section{Summary}

Ultimately, there is as of yet no single, magical plasmonic material, ${ }^{141}$ even in the reduced design space of the mid-IR. The choice of a plasmonic material is highly application specific, with limitations imposed by material compatibility, price, fabrication processes, and implementation environment. At the same time, significant consideration must be paid to material choice in order to meet the specifications of a given implementation. For this reason, we have attempted, in this work, to provide an overview of the wide range of materials available for plasmonic applications at mid-IR wavelengths. In particular, we have considered traditional plasmonic materials (the noble metals) as well as newer material systems with IR/VIS plasma wavelengths (transition metal nitrides, transparent conducting oxides, and silicides). However, applications benefitting from strong field confinement in the mid-IR require materials with mid-IR plasma wavelengths, and to this end, we discussed not only doped semiconductors (both III-V and group IV) but also materials with phononic resonances in the mid-IR, such as $\mathrm{GaN}, \mathrm{GaP}, \mathrm{SiC}$, and the perovskite $\mathrm{SrTiO}_{3}$. In the end, the broad nature of potential mid-IR plasmonic applications ensures that all of the discussed materials may have a role to play in the expanding field of mid-IR photonics.

\section{Acknowledgments}

D.W. would like to acknowledge support from the National Science Foundation, Award ECCS 14-20952. S.M. gratefully acknowledges support from the Joan and Lalit Bahl Fellowship from the Illinois ECE Department.

\section{References}

1. J. N. Anker et al., "Biosensing with plasmonic nanosensors," Nat. Mater. 7, 442-453 (2008).

2. J. Homola, "Surface plasmon resonance sensors for detection of chemical and biological species," Chem. Rev. 108, 462-493 (2008).

3. http://www.bionavis.com/products/ (14 January 2015).

4. N. Zhou et al., "Plasmonic near-field transducer for heat-assisted magnetic recording," Nanophotonics 3(3), 141-155 (2014).

5. A. M. Gobin et al., "Near-infrared resonant nanoshells for combined optical imaging and photothermal cancer therapy," Nano Lett. 7(7), 1929-1934 (2007).

6. O. Neumann et al., "Solar vapor generation enabled by nanoparticles," ACS Nano 7, 42-49 (2013).

7. A. Boltasseva and H. A. Atwater, "Low-loss plasmonic metamaterials," Science 331, 290 (2011).

8. J. B. Khurgin and A. B. Boltasseva, "Reflecting upon the losses in plasmonics and metamaterials," MRS Bull. 37, 768-779 (2012).

9. Y.-J. Lu et al., "Plasmonic nanolaser using epitaxially grown silver film," Science 337, 450 (2012).

10. K. Seal et al., "Coexistence of localized and delocalized surface plasmon modes in percolating metal films," Phys. Rev. Lett. 97, 206103 (2006).

11. F. M. Hoffman, "Infrared reflection-absorption spectroscopy of adsorbed molecules," Surf. Sci. Rep. 3, 107-192 (1983).

12. M. Osawa, "Dynamic processes in electrochemical reactions studied by surface-enhanced infrared absorption spectroscopy (SEIRAS)," Bull. Chem. Soc. Jpn. 70, 2861-2880 (1997).

13. P. B. Johnson and R. W. Christie, "Optical constants of the noble metals," Phys. Rev. B 6, 4370-4379 (1972).

14. M. A. Ordal et al., "Optical properties of the metals $\mathrm{Al}, \mathrm{Co}, \mathrm{Cu}, \mathrm{Au}, \mathrm{Fe}, \mathrm{Pb}, \mathrm{Ni}, \mathrm{Pd}, \mathrm{Pt}, \mathrm{Ag}$, Ti, and W in the infrared and far infrared," Appl. Opt. 22, 1099-1119 (1983).

15. A. I. Golovashkin et al., "Optical properties of Indium," Sov. Phys. JETP 24, 1093-1100 (1967). 
16. E. J. Zeman and G. C. Shatz, "An accurate electromagnetic theory study of surface enhancement factors for silver, gold, copper, lithium, sodium, aluminum, gallium, indium, zinc, and cadmium," J. Phys. Chem. 91(3), 634-643 (1987).

17. O. Hunderi and R. Ryberg, "Amorphous gallium-a free electron metal," J. Phys. F 4, 2096-2102 (1974).

18. A. D. Rakić et al., "Optical properties of metallic films for vertical-cavity optoelectronic devices," Appl. Opt. 37, 5271-5283 (1998).

19. K. Maex, "Silicides for integrated circuits: TiSi ${ }_{2}$ and CoSi2," Mater. Sci. Eng. R11, 53-153 (1993).

20. S. P. Murarka, Silicides for VLSI Applications, Academic Press, New York (1983).

21. L. Krusin-Elbaum and M. O. Aboelfotoh, "Unusually low resistivity of copper germanide thin films formed at low temperatures," Appl. Phys. Lett. 58, 1341 (1991).

22. J. W. Cleary et al., "IR permittivities for silicides and doped silicon," J. Opt. Soc. Am. B 27, 730-734 (2010).

23. M. G. Blaber, M. D. Arnold, and M. J. Ford, "Designing materials for plasmonic systems: the alkali-noble intermetallics," J. Phys. 22, 095501 (2010).

24. G. V. Naik, J. Kim, and A. Boltasseva, "Oxides and nitrides as alternative plasmonic materials in the optical range," Opt. Mater. Express 1, 1090-1099 (2011).

25. G. V. Naik et al., "Demonstration of $\mathrm{Al}$ : $\mathrm{ZnO}$ as a plasmonic component for near-infrared metamaterials," Proc. Natl. Acad. Sci. USA 109, 8834-8838 (2012).

26. G. V. Naik et al., "Titanium nitride as a plasmonic material for visible and near-infrared wavelengths," Opt. Mater. Express 2, 478-489 (2012).

27. D. S. Ginley and C. Bright, "Transparent conducting oxides," MRS Bull. 25, 15-18 (2000).

28. A. Kildishev and V. Shalaev, "Engineering space for light via transformation optics," Opt. Lett. 33, 43 (2008).

29. D. H. Ehlers and D. L. Mills, "Surface plasmons on n-type semiconductors: influence of depletion and accumulation layers," Phys. Rev. B 36, 1051 (1987).

30. F. Marquier et al., "Engineering infrared emission properties of silicon in the near field and the far field," Opt. Commun. 237, 379 (2004).

31. J. Gómez Rivas et al., "Propagation of surface plasmon polaritons on semiconductor gratings," Phys. Rev. Lett. 93, 256804 (2004).

32. M. Shahzad et al., "Infrared surface plasmons on heavily doped silicon," J. Appl. Phys. 110, 123105 (2011).

33. T. S. Moss, "The interpretation of the properties of indium arsenide," Proc. Phys. Soc. B 67(10), 775-782 (1954).

34. E. Burstein, “Anomalous optical absorption limit in InSb," Phys. Rev. 93(3), 632-633 (1954).

35. S. Law et al., "Mid-infrared designer metals," Opt. Express 20, 12155 (2012).

36. S. Law, R. Liu, and D. Wasserman, "Doped semiconductors with band-edge plasma frequencies," J. Vac. Sci. Technol. B 32(5), 052601 (2014).

37. S. Law, L. Yu, and D. Wasserman, "Epitaxial growth of engineered metals for mid-infrared plasmonics," J. Vac. Sci. Technol. B 31(3), 03C121 (2013).

38. W. Streyer et al., "Strong absorption and selective emission from engineered metals with dielectric coatings," Opt. Express 21, 9113-9122 (2013).

39. A. Rosenberg et al., "Flat mid-infrared composite plasmonic materials using lateral doping-patterned semiconductors," J. Opt. 16, 094012 (2014).

40. J. C. Ginn et al., "Infrared plasmons on heavily-doped silicon," J. Appl. Phys. 110, 043110 (2011).

41. J. Le Gall, M. Olivier, and J.-J. Greffet, "Experimental and theoretical study of reflection and coherent thermal emission by a SiC grating supporting a surface-phonon polariton," Phys. Rev. B 55, 10105 (1997).

42. S. C. Kehr et al., "Near field examination of perovskite-based superlenses and superlensenhanced probe-object coupling," Nat. Commun. 2(249), 1-9 (2011).

43. H. Harima, H. Sakashita, and S. Nakashima, "Raman microprobe measurement of under-damped LO-phonon-plasmon coupled mode in n-Type GaN," Mat. Sci. Forum 1363, 264-268 (1998). 
44. A. S. Barker, Jr., "Dielectric dispersion and phonon line shape in gallium phosphide," Phys. Rev. 165, 917 (1968).

45. W. Streyer et al., "Engineering absorption and blackbody radiation in the far-infrared with surface phonon polaritons on gallium phosphide," Appl. Phys. Lett. 104(13), 131105 (2014).

46. M. Silveirinha and N. Engheta, "Tunneling of electromagnetic energy through subwavelength channels and bends using $\varepsilon$-near zero materials," Phys. Rev. Lett. 97(15), 157403 (2006).

47. K. Halterman and S. Feng, "Resonant transmission of electromagnetic fields through subwavelength zero- $\varepsilon$ slits," Phys. Rev. A 78, 021805 (2008).

48. A. Alù et al., "Epsilon-near-zero metamaterials and electromagnetic sources: Tailoring the radiation phase pattern," Phys. Rev. B 75, 155410 (2007).

49. R. Ziolkowski, "Propagation in and scattering from a matched metamaterial having a zero index of refraction," Phys. Rev. E 70, 046608 (2004).

50. D. C. Adams et al., "Funneling light through a subwavelength aperture with epsilon-nearzero materials," Phys. Rev. Lett. 107(13), 133901 (2011).

51. S. Inampudi et al., " $\varepsilon$-near-zero enhanced light transmission through a subwavelength slit," Phys. Rev. B 89, 125119 (2014).

52. M. I. Stockman, "Nanoplasmonics: the physics behind the applications," Phys. Today 64, 39-44 (2011).

53. S. Underwood and P. Mulvaney, "Effect of the solution refractive index on the color of gold colloids," Langmuir 10(10), 3427-3430 (1994).

54. S. Link and M. A. El-Sayed, "Spectral properties and relaxation dynamics of surface plasmon electronic oscillations in gold and silver nano-dots and nano-rods," J. Phys. Chem. B 103, 8410-8426 (1999).

55. J. Perez-Juste et al., "Gold nanorods: synthesis, characterization and applications," Coord. Chem. Rev. 249, 1870-1901 (2005).

56. L. Novotny, "Effective wavelength scaling for optical antennas," Phys. Rev. Lett. 98, 266802 (2007).

57. R. Adato et al., "Ultra-sensitive vibrational spectroscopy of protein monolayers with plasmonic nanoantenna arrays," Proc. Natl. Acad. Sci. USA 106(46), 19227-19232 (2009).

58. F. Neubrech et al., "Resonant plasmonic and vibrational coupling in a tailored nanoantenna for infrared detection," Phys. Rev. Lett. 101, 157403 (2008).

59. R. L. Olman et al., "Determination of electric-field, magnetic field, and electric-current distribution of infrared optical antennas: a near-field optical vector network analyzer,' Phys. Rev. Lett. 105, 167403 (2010).

60. S. Law, V. Podolskiy, and D. Wasserman, "Towards nano-scale photonics with micro-scale photons: the opportunities and challenges of mid-infrared plasmonics," Nanophotonics 2, 104 (2013).

61. S. Law et al., "All-semiconductor plasmonic nanoantennas for infrared sensing," Nano Lett. 13, 4560 (2013).

62. R. Bardhan et al., "Nanosphere in a nanoshell: a simple nanomatryshka," J. Phys. Chem. C 114, 7378 (2010).

63. E. Ringe et al., "Unraveling the effects of size, composition, and substrate on the localized surface plasmon resonance frequencies of gold and silver nanocubes: a systematic singleparticle approach," J. Phys. Chem. C, 114(29), 12511-12516 (2010).

64. N. Verellen et al., "Plasmon line shaping using nanocrosses for high sensitivity localized surface plasmon resonance sensing," Nano Lett. 11, 391-397 (2011).

65. S. Link and M. A. El-Sayed, "Size and temperature dependence of the plasmon absorption of colloidal gold nanoparticles," J. Phys. Chem. B 103, $4212-4217$ (1999).

66. L. J. Sherry et al., "Localized surface plasmon resonance spectroscopy of single silver triangular nanoprisms," Nano Lett. 6(9), 2060-2065 (2006).

67. M. G. Blaber et al., "Plasmon absorption in nanospheres: a comparison of sodium, potassium, aluminum, silver, and gold," Physica B 394, 184-187 (2007).

68. F. Wang and Y. R. Shen, "General properties of local plasmons in metal nanostructures," Phys. Rev. Lett. 97, 206806 (2006). 
69. M. D. Arnold and M. G. Blaber, "Optical performance and metallic absorption in nanoplasmonic systems," Opt. Express 17, 3835-3847 (2009).

70. J. D. Jackson, Classical Electrodynamics, John Wiley and Sons, New York (1975).

71. G. V. Naik, V. M. Shalev, and A. Boltasseva, "Alternative plasmonic materials: beyond gold and silver," Adv. Mater. 25, 3264-3294 (2013).

72. P. Berini, "Long-range surface plasmon polaritons," Adv. Opt. Photonics 1, 484-588 (2009).

73. M. Hill et al., "Lasing in metal-insulator-metal sub-wavelength plasmonic waveguides," Opt. Express 17, 11107-11112 (2009).

74. J. A. Dionne et al., "PlasMOStor: a metal-oxide-silicon field-effect plasmonic modulator," Nano-Lett. 9, 897-902 (2006).

75. R. F. Oulton et al., "Plasmon lasers at deep subwavelength scale," Nature 461, 629-632 (2009).

76. J. A. Dionne, H. J. Lezec, and H. A. Atwater, "Highly confined photon transport in subwavelength metallic slot waveguides," Nano Lett. 6, 1928-1932 (2006).

77. G. Veronis and S. Fan, "Modes of subwavelength plasmonic slot waveguides," J. Lightwave Technol. 25, 2511-2521 (2007).

78. G. Veronis and S. Fan, "Guided subwavelength plasmonic mode supported by a slot in a thin metal film," Opt. Lett. 30, 3359-3361 (2005).

79. J. A. Dionne, L. A. Sweatlock, and H. A. Atwater, "Plasmon slot waveguides: Towards chip-scale propagation with subwavelength-scale localization," Phy. Rev. B 73, 035407 (2006).

80. J. Liu et al., "Surface plasmon reflector based on serial stub structure," Opt. Express 17, 20134-20139 (2009).

81. S. A. Maier et al., "Local detection of electromagnetic energy transport below the diffraction limit in metal nanoparticle plasmon waveguides," Nat. Mater. 2, 229-232 (2003).

82. W. L. Barnes, "Surface plasmon-polariton length scales: a route to sub-wavelength optics," J. Opt. A: Pure Appl. Opt. 8, S87-S93 (2006).

83. W. L. Barnes, A. Dereux, and T. W. Ebbesen, "Surface plasmon subwavelength optics," Nature 424, 824 (2003).

84. T. W. Ebbesen, C. Genet, and S. I. Bozhevolnyi, "Surface-plasmon circuitry," Phys. Today 61, 44-50 (2008).

85. S. A. Maier, Plasmonics: Fundamentals and Applications, Springer, New York (2007).

86. P. Berini, "Figures of merit for surface plasmon waveguides," Opt. Express 14, 13030 13042 (2006).

87. P. Berini, "Plasmon-polariton waves guided by thin lossy metal films of finite width: bound modes of symmetric structures," Phys. Rev. B 6110484 (2000).

88. R. Zia et al., "Geometries and materials for subwavelength surface plasmon modes," J. Opt. Soc. Am. A 21, 2442-2446 (2004).

89. R. F. Oulton et al., "Confinement and propagation characteristics of subwavelength plasmonic modes," New J. Phys. 10, 105018 (2008).

90. R. Buckley and P. Berini, "Figures of merit for 2D surface plasmon waveguides and application to metal stripes," Opt. Express 15(19), 12174 (2007).

91. S. A. Maier and H. A. Atwater, "Plasmonics: localization and guiding of electromagnetic energy in metal/dielectric structures," J. Appl. Phys. 98, 011101 (2005).

92. S. A. Maier, "Effective mode volume of nanoscale plasmon cavities," Opt. Quant. Electron. 38, 257-267 (2006).

93. A. V. Krasavin and A. V. Zayats, "Guiding light at the nanoscale: numerical optimization of ultrasubwavelength metallic wire plasmonic waveguides,” Opt. Lett. 36, 3127-3129 (2011).

94. K. S. Novoselov et al., "Electric field effect in atomically thin carbon films," Science 306, 666 (2004).

95. K. S. Novoselov et al., "Two-dimensional atomic crystals," Proc. Natl. Acad. Sci. USA 102, 10451 (2005). 
96. K. S. Novoselov et al., "Two-dimensional gas of massless Dirac fermions in graphene," Nature 438, 197-200 (2005).

97. S. J. Allen, D. C. Tsui, and R. A. Logan, "Observation of the two-dimensional plasmon in silicon inversion layers," Phys. Rev. Lett. 38, 980 (1977).

98. E. A. Shaner et al., "Single-quantum-well grating-gated terahertz plasmon detectors," Appl. Phys. Lett. 87, 193507 (2005).

99. F. H. L. Koppens, D. E. Chang, and F. J. G. de Abajo, "Graphene plasmonics: a platform for strong light-matter interactions," Nano Lett. 11, 3370 (2011).

100. A. N. Grigorenko, M. Polini, and K. S. Novoselov, "Graphene plasmonics," Nat. Photonics 6, 749-758 (2012).

101. F. J. G. de Abajo, "Graphene plasmonics: challenges and opportunities," ACS Photonics 1, 135-152 (2014).

102. W. Feng et al., "Gate-variable optical transitions in graphene," Science 320, 206 (2008).

103. Y.-J. Yu et al., "Tuning the graphene work function by electric field effect," Nano Lett. 9(10), 3430-3434 (2009).

104. L. S. Panchakarla et al., "Synthesis, structure, and properties of boron- and nitrogen-doped graphene," Adv. Mater. 21, 4726-4730 (2009).

105. H. Liu, Y. Liu, and D. Zhua, "Chemical doping of graphene," J. Mater. Chem. 21, 33353345 (2011).

106. A. Das et al., "Monitoring dopants by Raman scattering in an electrochemically top-gated graphene transistor," Nat. Nanotech. 3, 210-215 (2008).

107. M. Jablan, H. Buljan, and M. Soljacic, "Plasmonics in graphene at infrared frequencies," Phys. Rev. B 80, 245435 (2009).

108. C.-F. Chen et al., "Controlling inelastic light scattering quantum pathways in graphene," Nature 471, 617-620 (2011).

109. Y.-C. Chang et al., "Extracting the complex optical conductivity of mono- and bilayer graphene by ellipsometry," Appl. Phys. Lett. 104, 261909 (2014).

110. T. Low and P. Avouris, "Graphene plasmonics for terahertz to mid-infrared applications," ACS Nano 8(2), 1086-1101 (2014).

111. K. F. Mek et al., "Measurement of the optical conductivity of graphene," Phy. Rev. Lett. 101, 196405 (2008).

112. Z. Fei et al., "Gate-tuning of graphene plasmons revealed by infrared nano-imaging," Nature 487, 82-85 (2012).

113. J. Chen et al., "Optical nano-imaging of gate-tunable graphene plasmons," Nature 487, 77-81 (2012).

114. B. Vasic, G. Isic, and R. Gajic, "Localized surface plasmon resonances in graphene ribbon arrays for sensing of dielectric environment at infrared frequencies," J. Appl. Phys. 113, 013110 (2013).

115. H. Yan et al., "Damping pathways of mid-infrared plasmons in graphene nanostructures," Nat. Photonics 7, 394-399 (2013).

116. F. Xia et al., "Two-dimensional material nanophotonics," Nature Photon. 8, 899-907 (2014).

117. H. Chu and C. H. Gan, "Active plasmonic switching at mid-infrared wavelengths with graphene ribbon arrays," Appl. Phys. Lett. 102, 231107 (2013).

118. H. Yan et al., "Tunable infrared plasmonic devices using graphene/insulator stacks," Nature Nanotechnol. 7, 330-334 (2012).

119. Z. Fang et al., "Active tunable absorption enhancement with graphene nanodisk arrays," Nano Lett. 14, 299-304 (2014).

120. L. Ju et al., "Graphene plasmonics for tunable terahertz metamaterials," Nat. Nanotechnol. 6, 630-634 (2011)

121. J.-J. Greffet et al., "Coherent emission of light by thermal sources," Nature 416, 61-64 (2002).

122. J. Faist et al., "Quantum cascade laser," Science 264, 553 (1994).

123. Y. Yao, A. J. Hoffman, and C. F. Gmachl, "Mid-infrared quantum cascade lasers," Nat. Photonics 6(7), 432-439 (2012). 
124. R. Q. Yang et al., "Mid-infrared type-II interband cascade lasers," Quantum Electron. 38(6), 559-568 (2002).

125. W. W. Bewley et al., "Continuous-wave interband cascade lasers operating above room temperature at $\lambda=4.7-5.6 \mu \mathrm{m}$," Opt. Express 20(3), 3235-3240 (2012).

126. B. F. Levine, "Quantum-well infrared photodetectors," J. Appl. Phys. 74, R1 (1993).

127. K. W. Berryman, S. A. Lyon, and M. Segev, "Mid-infrared photoconductivity in InAs quantum dots," Appl. Phys. Lett. 70, 1861 (1997).

128. D. L. Smith and C. Mailhiot, "Proposal for strained type II superlattice infrared detectors," J. Appl. Phys. 62, 2545 (1987).

129. S. Maimon and G. W. Wicks, "nBn detector, an infrared detector with reduced dark current and higher operating temperature," Appl. Phys. Lett, 89, 151109 (2006).

130. Z. Yu et al., "Design of midinfrared photodetectors enhanced by surface plasmons on grating structures," Appl. Phys. Lett. 89, 151116 (2006).

131. X. Hu et al., "Design of midinfrared photodetectors enhanced by resonant cavitities with subwavelength metallic gratings," Appl. Phys. Lett. 93, 241108 (2008).

132. W. Wu, A. Bonakdar, and H. Mohseni, "Plasmonic enhanced quantum well infrared photodetector with high detectivity," Appl. Phys. Lett. 96, 161107 (2010).

133. S. C. Lee, S. Krishna, and S. R. J. Brueck, "Quantum dot infrared photodetector enhanced by surface plasma wave excitation," Opt. Express 17, 23160-23168 (2009).

134. J. Rosenberg et al., "A multispectral and polarization-selective surface plasmon resonant midinfrared detector," Appl. Phys. Lett. 95, 161101 (2009).

135. C. Y. Chang et al., "Wavelength selective quantum dot infrared photodetector with periodic metal hole arrays," Appl. Phys. Lett. 91, 163107 (2007).

136. S. J. Lee et al., "A monolithically integrated plasmonic infrared quantum dot camera," Nat. Commun. 2, 286 (2011).

137. C. Sirtori et al., "Long-wavelength $(\lambda \approx 8-11.5 \mu \mathrm{m})$ semiconductor lasers with waveguides based on surface plasmons," Opt. Lett. 23, 1366-1368 (1998).

138. A. Babuty et al., "Semiconductor surface plasmon sources," Phys. Rev. Lett. 104, 226806 (2010).

139. S. Law et al., "All-semiconductor plasmonic perfect absorbers," Phys. Rev. Lett. 112, 017401 (2014).

140. D. Li and C. Z. Ning, "All-semiconductor active plasmonic system in mid-infrared wavelengths," Opt. Express 19, 14594-14603 (2011).

141. J. B. Khurgin and G. Sun, "In search of the elusive lossless metal," Appl. Phys. Lett. 96, 181102 (2010).

Yujun Zhong is now a postdoctoral researcher at the University of Illinois at UrbanaChampaign. She received her $\mathrm{PhD}$ degree in materials science and engineering from the University of Delaware and a BS degree in optical information, science, and technology from Sun Yat-sen University (China). She currently studies plasmonic and photonic materials using molecular beam epitaxial (MBE) growth. She is interested in III-V semiconductors, plasmonic materials, IR optoelectronics, photovoltaics, and thermoelectrics.

Daniel Wasserman is an assistant professor at the University of Illinois's Department of Electrical Engineering. His research focuses on plasmonic and metamaterial devices and structures, nanotechnology, and semiconductor-based material systems for mid-IR wavelengths. He received his ScB from Brown University (1998) and his PhD from Princeton University (2004). At Princeton, he was a Francis Upton Fellow and a NSF Graduate Fellow. He is the recipient of NSF CAREER and AFOSR Young Investigator Awards.

Biographies of the other authors are not available. 